\title{
Acidic microenvironment shaped by lactate accumulation promotes pluripotency through multiple mechanisms
}

Authors: Wen-Ting Guo ${ }^{1,2}$, Shao-Hua Wang², Xiao-Shan Zhang², Ming Shi², Jing Hao², Xi-Wen Wang ${ }^{2}$, Kai-Li Gu², Fei-Fei Duan², Ying Yan², Xi Yang², Chao Zhang², Le-Qi Liao², Yangming Wang $^{2, *}$

\section{Affiliations:}

1 Central laboratory, Peking Union Medical College Hospital, Peking Union Medical College and Chinese Academy of Medical Sciences, No.1 Shuaifuyuan, Beijing, 100730, P.R. China.

2 Beijing Key Laboratory of Cardiometabolic Molecular Medicine, Institute of Molecular Medicine, Peking University, Beijing 100871, China

*To whom correspondence should be addressed to. Tel: +8610-62766945; Fax: +8610-62766945;

Email: yangming.wang @pku.edu.cn (YW)

Running title: Acidic microenvironment promotes pluripotency 


\begin{abstract}
Enhanced glycolysis is a distinct feature associated with numerous stem cells and cancer cells. However, little is known about its regulatory roles in gene expression and cell fate determination. Here we show that acidic environment shaped by lactate accumulation promotes the self-renewal and pluripotency of both mouse and human embryonic stem cells (ESCs). Mechanistically, acidic $\mathrm{pH}$ reduces the tri-methylation of H3K27 globally at transcriptional start sites to partially prevent ESC differentiation. In addition, acidic $\mathrm{pH}$ stabilizes a large number of mRNAs including pluripotency genes. Furthermore, we found that AGO1 protein is downregulated at acidic conditions, leading to the de-repression of a subset of microRNA targets in low-pH treated ESCs. Altogether, our study provides insights into mechanisms whereby acidic microenvironment produced by enhanced glycolysis regulates gene expression to determine cell fate and has broad implications in the fields of regenerative medicine and cancer biology.
\end{abstract}




\section{Introduction}

All cells require energy to maintain a high degree of order for survival and physiological functions. For animals the energy is derived from food molecules by wellorganized catalytic processes degrading organic molecules such as proteins, lipids and polysaccharides. Two fundamental metabolic processes generating energy are glycolysis and oxidative phosphorylation (OXOPHOS). While glycolysis generates only 2 ATP molecules per glucose, OXOPHOS generates 38 ATP molecules per glucose. During OXOPHOS glucose is completely oxidized into $\mathrm{CO}_{2}$ and $\mathrm{H}_{2} \mathrm{O}$. In contrast, glycolysis provides intermediate metabolites for various anabolic processes to synthesize nucleotides, lipids and amino acids ${ }^{1,2}$. Interestingly, previous studies have shown that cancer cells and pluripotent stem cells (PSCs) prefer glycolysis rather than more efficient OXOPHOS for energy supply even in the presence of sufficient oxygen ${ }^{3-}$ 5 . Likewise, during reprogramming, the acquisition of pluripotency is associated with the shift from oxidative to glycolytic metabolism. In contrast, during ESC differentiation, decreased glycolysis is observed with the energy supply of cells becoming increasingly dependent on $\mathrm{OXOPHOS}^{6,7}$. In unicellular organisms, the metabolism switch is made possible simply by responding to the abundance of nutrient supplies in the environment. However, due to sufficient and constant nutrient supplies at most time, the metabolism switch of cells in multiple cellular organisms like mammals is regulated by sophisticated genetic circuits. Specifically in PSCs, various pluripotency-associated genes including $\mathrm{Myc}^{8}{ }^{9}$, Esrrb and $\mathrm{Zic} 3^{10}$, and miRNAs including miR-290 and miR-200 families ${ }^{11,12}$ have been shown to regulate their preference for glycolysis over OXOPHOS. Additionally, Hifla is shown to promote the switch from bivalent to exclusively glycolytic metabolism during ESC to EpiSC transition ${ }^{13}$. These findings strongly suggest the importance of metabolism regulation in PSCs. However, the functional role and the working mechanism of different metabolic pathways in cell fate determination are still at large.

PSCs can undergo rapid self-renewal while retaining the ability to differentiate into all 
somatic cells in the body. Understandably, rapid proliferation requires the synthesis of a large quantity of biomolecules. For this reason, glycolysis has been proposed to provide abundant anabolic intermediates to support rapid proliferation ${ }^{1,2}$. Furthermore, recent studies demonstrate that glycolysis play functional roles in keeping PSCs undifferentiated $^{9,14}$. The exact mechanism by glycolysis to promote self-renewal and pluripotency is not well understood. Chromatin modification is one of the most important processes controlling the self-renewal and pluripotency of $\mathrm{PSCs}^{15,} 16$. Chromatin modifiers require various cofactors and substrates for the chemical reaction of modification processes. Recent studies found that alpha-ketoglutarate and acetate generated from glucose flux are essential for histone methylation and acetylation by acting as a cofactor for histone demethylases ${ }^{17}$ and a substrate for histone acetyltransferases ${ }^{14}$, respectively. These studies reveal important functions of intermediate metabolites from glycolysis in regulating epigenetic modifications in PSCs. Nevertheless, besides many intermediate metabolites, glycolysis also produces a large amount of lactate which is transported into the extracellular space of PSCs. In fact, PSCs convert more than half the glucose consumed to lactate in vivo and in vitro $9,18,19$. It is estimated that the concentration of lactate can reach $16 \mathrm{mM}$ and $100 \mathrm{mM}$ in mouse and human blastocyst over a $24-\mathrm{hr}_{\text {period }}{ }^{20}$, respectively. Hence, aerobic glycolysis can generate a very high concentration of lactate to alter the microenvironment of PSCs in vivo. Functionally, acidic microenvironment has been reported to enhance invasive and angiogenic potential of tumor cells $\mathrm{s}^{21-23}$. However, the function of acidic $\mathrm{pH}$ has not been thoroughly investigated in PSCs. Recently, through inhibiting $\mathrm{Na}^{+}-\mathrm{H}^{+}$exchanger DNhe2 (fly) or NHE1 (mouse), Ulmschneider et al found that the increase of intracellular $\mathrm{pH}$ is important for the differentiation of adult fly follicle stem cells and mouse $\mathrm{ESCs}^{24}$, implicating the function of lactic acid in regulating the self-renewal and pluripotency of stem cells.

In this study, we show that acidic microenvironment shaped by lactate accumulation play important functions in sustaining the self-renewal and pluripotency of PSCs. Lowering $\mathrm{pH}$ of culture media below 7 is sufficient to maintain the self-renewal and 
pluripotency of both mouse and human ESCs in normally differentiation conditions. Mouse ESCs can be maintained at pluripotent state for long term in basal media containing only LIF at low $\mathrm{pH}$, replacing the requirement for chemical inhibitors to GSK3 and MEK. Mechanistically, we showed that low pH worked through reducing the trimethylation of $\mathrm{H} 3 \mathrm{~K} 27$ at transcriptional start sites, stabilizing mRNAs of pluripotency genes and suppressing miRNA activities. Together, these findings significantly improve our understanding of how acidic microenvironment produced by enhanced glycolysis regulates gene expression to control cell fate and have broad implications for the fields of regenerative medicine and cancer biology.

\section{Results}

\section{Lactic acid but not lactate promotes the self-renewal and pluripotency of mouse ESCs}

To monitor ESC differentiation process, we constructed mESCs expressing d2EGFP under the control of Rex 1 promoter. The expression of GFP correlates with the activity of Rex 1 promoter and therefore the pluripotency state of $\mathrm{ESCs}^{25}$. Rex1-d2EGFP ESCs expressed high levels of GFP when cultured in $2 \mathrm{i}+\mathrm{LIF}$ media. However, they almost completely lost the expression of GFP when cultured in basal media without $2 \mathrm{i}$ and LIF for around 2 days (Fig. 1a, b). To investigate the influence of lactate generated by glycolysis on the pluripotency of ESCs, we supplemented basal media with $10 \mathrm{mM}$ lactic acid or lactate salt. Surprisingly, lactic acid but not lactate sustained high levels of Rex1-d2EGFP expression (Fig. 1a, b). These data suggest that extracellular acidity caused by glycolysis is sufficient to support the pluripotency state of mouse ESCs for at least a short period. The addition of $10 \mathrm{mM}$ lactic acid lowers the $\mathrm{pH}$ of basal media from $\sim 7.4$ to $\sim 6.8$. Consistent with acidic environment but not lactate salt promoting the self-renewal and pluripotency of ESCs, adjusting $\mathrm{pH}$ to 6.8 by other inorganic acids, including hydrochloride acid and sulphuric acid, also blocked the loss of Rex1-d2EGFP expression in the absence of $2 \mathrm{i}$ and LIF (Fig. 1c).

To test what $\mathrm{pH}$ range provides the best effect, we grew Oct4-GFP-ires-Puro ESCs in 
differentiation media under various $\mathrm{pH}$ conditions and then evaluated for the maintenance of pluripotency by their ability to proliferate in $2 \mathrm{i}$ culture plus puromycin resistance ${ }^{26}$. The results show that acidic but not basic conditions promoted the selfrenewal and pluripotency of ESCs with $\mathrm{pH}$ 6.0-6.4 being the most efficient (Supplementary Fig. 1a). Culturing ESCs at pH 5.5 or lower led to extensive cell death, making it impossible to evaluate their effects on pluripotency. We then further verified that $\mathrm{pH} 6.4$ blocked the exit of pluripotency by flow cytometry analysis of Rex1d2EGFP levels (Supplementary Fig. 1b, c) and qRT-PCR analysis of pluripotency and differentiation markers (Fig. 1d). Consistent with these results, NANOG protein was also highly expressed in N2B27-pH6.4 condition (Fig. 1e). Furthermore, ESCs cultured in N2B27-pH 6.4 retained a compact colony morphology, which indicates an undifferentiated state (Fig. 1f). Likewise, colony formation assay also confirmed the blocking of differentiation by pH 6.4 (Fig. 1g, h). To check the generality of acidic media blocking differentiation, we performed similar experiments in conventional serum media. Consistently, RT-PCR analysis showed that acidic condition blocked the differentiation of ESCs in serum media without LIF supplement as well (Supplementary Fig. 1d). Together, these results show that acidic microenvironment due to the accumulation of lactic acid promotes the self-renewal and pluripotency of mouse ESCs.

To test whether endogenously produced lactate is sufficient to support the self-renewal and pluripotency of mouse ESCs, we grew ESCs in serum media with $1 \mu \mathrm{M}$ retinoid acid (RA) with or without HEPES buffer. After growing for approximately 48 hours, the $\mathrm{pH}$ of unbuffered media is decreased from 7.4 to 6.3 (Supplementary Fig. 1e), suggesting endogenously produced lactate sufficient to change the $\mathrm{pH}$ of extracellular environment. In contrast, the $\mathrm{pH}$ of buffered media remained constantly at 7.4. Consistent with $\mathrm{pH}$ changes, qPCR assay showed that ESCs cultured in unbuffered media retained high expression of pluripotency markers while ESCs in buffered media are differentiated (Figure 1i). Altogether, these data show that glycolysis can produce sufficient amount of lactate to block ESC differentiation. 
Transcriptomic and epigenomic analyses confirm that acidic condition maintains the pluripotency of ESCs

To fully characterize ESCs cultured in acidic media, we performed RNA-Seq, small RNA-Seq and ChIP-Seq using antibodies against OCT4, H3K27 tri-methylation (H3K27me3) and H3K4 tri-methylation (H3K4me3) for ESCs cultured in 2i+LIF-pH 7.4, 2i+LIF-pH 6.4, N2B27-pH 7.4 and N2B27-pH6.4. Principle component analysis (PCA) and unsupervised hierarchical clustering analysis of transcriptome (Fig. 2a, Supplementary Fig. 2a, b) and miRNA profiles (Fig. 2b, Supplementary Fig. 2c) showed that N2B27-pH 6.4 cultured ESCs clustered closer to $\mathrm{pH} 7.4$ and pH 6.4 2i+LIF ESCs and are evidently separated from differentiated ESCs in N2B27-pH 7.4. As expected, low $\mathrm{pH}$ significantly inhibited the expression of differentiation driving MEK and GSK3 pathway genes and promoted the expression of pluripotency supporting AKT and STAT3 pathway genes (Supplementary Fig. 2d). These data support that acidic $\mathrm{pH}$ maintains the self-renewal and pluripotency of ESCs.

Oct4 is one of the most important pluripotency regulators sitting at the center of the pluripotency transcriptional network ${ }^{27}$. We next asked whether Oct4 genomic occupancy was altered in N2B27-pH 6.4 ESCs. ChIP-Seq analyses showed that OCT4 binding remained high in N2B27-pH6.4 but is significantly downregulated in N2B27pH 7.4 at global level (Fig. 2c, d). Importantly, ChIP-qPCR and ChIP-seq analyses showed that low $\mathrm{pH}$ rescued OCT4 binding at the promoter of many important pluripotency genes (Supplementary Fig. 2e, f). Furthermore, ChIP-seq analysis of $\mathrm{H} 3 \mathrm{~K} 27 \mathrm{me} 3$ showed a global upregulation of $\mathrm{H} 3 \mathrm{~K} 27 \mathrm{me} 3$ at transcriptional start sites in ESCs switched from 2i+LIF-pH7.4 to N2B27-pH 7.4 (Fig. 2e, f), consistent with substantial reorganization of $\mathrm{H} 3 \mathrm{~K} 27 \mathrm{me} 3$ markers during ESC differentiation ${ }^{28}$. As expected from cellular phenotypes and transcriptomic analyses, this trend was evidently blocked by low pH treatment (Fig. 2e, f and Supplementary Fig. 2g). In contrast, we did not observe any significant differences in H3K4me3 profiles between all four conditions (Fig. 2g, h). Altogether, these data show that low $\mathrm{pH}$ treated mESCs are essentially similar to naive mESCs cultured in $2 \mathrm{i}+\mathrm{LIF}$ at both transcriptomic and 
epigenomic levels.

\section{Low pH plus LIF maintain the long-term self-renewal of mouse ESCs}

We then tested whether basal media at $\mathrm{pH} 6.4$ can provide an alternative culture media to maintain and expand ESCs for long-term. Unfortunately, ESCs cultured in N2B27 at pH 6.4 could only be passaged for 2-3 passages and then quickly become degenerated (data not shown). Adding LIF to media helped to maintain ESC cultures for a few more passages. In addition, ESCs can be cultured in basal media at pH 6.8 with LIF for approximately one month, which were then gradually degenerated. These ESCs retained features of pluripotent ESCs, including compact colony morphologies (Supplementary Fig. 3a) and high expression of pluripotency markers (Supplementary Fig. 3b), while ESCs cultured in basal media at pH 7.4 plus LIF were mostly differentiated. These data demonstrate that basal media at $\mathrm{pH} 6.8$ plus LIF can maintain self-renewal and pluripotency of mESCs for a relatively long period.

\section{Low pH promotes the self-renewal and pluripotency of human ESCs}

Next, we tested whether low $\mathrm{pH}$ also promotes the self-renewal and pluripotency of human ESCs. Like mouse ESCs, human ESCs also heavily depend on aerobic glycolysis for energy supply ${ }^{9,13}$. Strikingly, pH 6.4 media without bFGF partially maintained the self-renewal of hESCs as gauged by cell morphology (Fig. 3a), and the expression of core pluripotency genes OCT4, SOX2 and NANOG (Fig. 3b). In addition, flow cytometry analysis of OCT4-GFP human ESCs showed that low pH maintained high activity of OCT4 promoter (Fig. 3c, d). Together, these results indicate that the pluripotency promoting function of acidic microenvironment is conserved for both mouse and human ESCs.

\section{Low pH cause global reduction of $\mathrm{H3K} 27$ trimethylation at transcription start sites}

Next, we sought to understand molecular mechanisms by acidic $\mathrm{pH}$ to promote the selfrenewal and pluripotency of ESCs. As shown in Figures 2c and 2d, we observed 
significant reduction of $\mathrm{H} 3 \mathrm{~K} 27$ tri-methylation at transcriptional start sites at low $\mathrm{pH}$ conditions. To assess the functional impact of $\mathrm{H} 3 \mathrm{~K} 27 \mathrm{me} 3$ reduction, we analyzed the expression of $\mathrm{H} 3 \mathrm{~K} 27 \mathrm{me} 3$ target genes and found they are significantly upregulated compared to all genes (Fig. 4a). In addition, for promoters that gained more H3K27me3 signals during differentiation, overall H3K27me3 intensity was significantly reduced in low $\mathrm{pH}$ cultures (Fig. $\mathbf{4 b}, \mathbf{c})$. Based on these data, we reasoned that if the reduction of $\mathrm{H} 3 \mathrm{~K} 27 \mathrm{me} 3$ is responsible for the blocking of differentiation at low $\mathrm{pH}$ conditions, inactivating Eed, a gene essential for the tri-methylation of H3K27, should also block the differentiation of ESCs. To test this hypothesis, we generated Eed knockout ESCs using CRISPR/Cas9 (Supplementary Fig. 4a-c). Interestingly, genes upregulated in Eed knockout ESCs were significantly enriched in low pH treated ESCs (Fig. 4d, e), suggesting that low $\mathrm{pH}$ upregulates gene expression at least partially through the reduction of the tri-methylation of H3K27. Importantly, compared to wild type ESCs, Eed knockout ESCs displayed higher expression of pluripotency genes in N2B27 differentiation conditions (Fig. 4f and Supplementary Fig. 4d). Nevertheless, the effect of blocking differentiation by knocking out Eed was not as significant as by low $\mathrm{pH}$ treatment. Taken together, these data show that acidic environment maintains the pluripotency and self-renewal of ESCs at least partially by reducing the tri-methylation of $\mathrm{H} 3 \mathrm{~K} 27$ at transcriptional start sites.

\section{Low pH stabilizes thousands of mRNAs including known pluripotency genes in ESCs}

Since knocking out Eed did not fully recapitulate the effects of low pH, we decided to search for other mechanisms by low $\mathrm{pH}$ in promoting the self-renewal and pluripotency of ESCs. Recent studies suggest that post-transcriptional regulation especially mRNA degradation is essential in controlling the pluripotency and differentiation of $\mathrm{ESCs}^{29-32}$. To check whether there is a link between low $\mathrm{pH}$ treatment and post-transcriptional regulation, we analyzed mRNA stability in low $\mathrm{pH}$ and control media. These experiments were performed in $2 \mathrm{i}+\mathrm{LIF}$ media in order to exclude any secondary effects due to differentiation. The mRNA life time profiling was performed by collecting and 
analyzing RNA-seq data obtained at 0,2, 4 and 8 hours after adding actinomycin $\mathrm{D}$, a potent inhibitor of RNA polymerase II (Fig. 5a). Interestingly, accumulation plot showed that mRNAs were globally stabilized in low pH treated cells (Fig. 5b). mRNA half-lives of 605 and 187 genes were increased or decreased by two fold (Fig. 5c), respectively. qRT-PCR analysis of randomly selected genes confirmed mRNA stabilization effects of low pH treatment (Fig. 5d and Supplementary Fig. 5a). As expected, overall expression level of stabilized genes and destabilized genes are upregulated and downregulated (Supplementary Fig. 5b, c), respectively.

We then analyzed whether mRNAs of pluripotency genes are stabilized. Dppa5a and Fbxo15 were slightly stabilized in 2i+LIF pH 6.4 versus 2i+LIF pH 7.4 ESCs (Fig. 5d). However, overall mRNA half-lives for pluripotency genes were similar in $2 \mathrm{i}+\mathrm{LIF}$ cells under normal or low-pH conditions (Fig. 5e). These results suggest: 1. low-pH has no effects in stabilizing pluripotency genes; or 2. low $\mathrm{pH}$ has effects in stabilizing pluripotency genes, but other mechanisms stabilizing pluripotency genes in $2 \mathrm{i}+\mathrm{LIF}$ condition cover up the function of low-pH treatment. If the latter is right, mRNA of pluripotency genes should become destabilized upon removing $2 \mathrm{i}+\mathrm{LIF}$ and low $\mathrm{pH}$ treatment should block this tendency. To test this, we analyzed mRNA half-life three hours after removing $2 \mathrm{i}$ and LIF, at which point N2B27-pH 7.4 cultured ESCs are still pluripotent. Interestingly, mRNA half-lives of 11 out of 12 selected pluripotency genes were significantly decreased, and low-pH stabilized 6 of them including Stat3, Pim1, Dppa5a, Ncoa3, Sox2 and Fbxo15 (Fig. 5f). Among them, overexpression of Stat3, Pim1 and Ncoa3 have been shown to block ESC differentiation in various cases ${ }^{33-35}$. These data suggest that low $\mathrm{pH}$ help maintain pluripotency state by stabilizing a subset of pluripotency mRNAs.

\section{Low pH stabilizes extracellular matrix genes in cancer cells}

To test whether stabilization of mRNAs is a general mechanism by low $\mathrm{pH}$ treatment in other cells, we measured mRNA half-life in U2OS cells cultured under normal and low $\mathrm{pH}$ media. Consistent with previous findings in cancer cells $\mathrm{s}^{23}, 36,37$, low-pH 
treatment promoted epithelial-mesenchymal transition (EMT) in U2OS and HepG2 cells (Supplementary Fig. 6a, b). In these cells, we did not observe similar global stabilization of transcripts as in ESCs (Fig. 5g), instead we found around 300 genes are stabilized or destabilized ( $>2$ fold changes of half life) upon low $\mathrm{pH}$ treatment. However, we found that mRNAs of extracellular matrix (ECM) proteins are significantly stabilized upon low-pH treatment (Fig. 5h and Supplementary Fig. 6c). This is consistent with EMT observed in these cells upon low pH treatment since upregulation of ECM components are associated with EMT in cancer cells. Therefore, low-pH treatment could stabilize mRNAs of a subset of key genes to promote EMT. Together, these data suggest that mRNA stabilization might be a general mechanism by low $\mathrm{pH}$ environment to confer functional consequences in ESCs and cancer cells.

\section{Low pH downregulates AGO1 protein expression and derepresses a subset of miRNA targets in ESCs}

miRNA is one of the most important post-transcriptional regulators that destabilizes mRNAs. To gain more insights on how low pH promotes ESC self-renewal and pluripotency, we considered the possibility that a subset of mRNAs are stabilized through the inhibition of miRNA activities. We focused on miR-294/302 family of miRNAs, which are the most enriched miRNAs in ESCs. We first defined their targets based on their 3'UTR sequences and their expression changes in $\operatorname{Dgcr} 8 \mathrm{KO}$ versus wild type ESCs (see Materials and Methods). Interestingly, we found mRNA targets of miR-294/302 family were significantly enriched in stabilized genes (Fig. 6a and Supplementary Fig. 7a). Consistently, GSEA showed that miR-294/302 targets are significantly upregulated in low pH treated ESCs (Fig. 6b and Supplementary Fig. 7b). RT-PCR confirmed the upregulation of previously verified miR-294 targets Fndc3b, Mbd2 and Tgfbr2 and the downregulation of Mbd2 target Myc (Fig. 6c). In addition, luciferase reporter assay showed that the inhibition at the 3'UTR of Mbd2 is partially reversed by low $\mathrm{pH}$ treatment (Fig. 6d). In the next, we verified that inhibition of miRNA activities indeed can significantly block ESC differentiation in the absence of $2 \mathrm{i}$ and LIF using $\operatorname{Dgcr} 8$ knockout ESC (Fig. 6e). These results are also consistent 
with our previous reports showing that miR-294/302 family is important for the efficient exit of naive pluripotency and subsequent differentiation of $\mathrm{ESCs}^{38,} 39$. Therefore, the de-repression of miRNA targets can partially explain the blocking of differentiation by low $\mathrm{pH}$ condition

The de-repression of miRNA targets could be due to the decrease of miRNA expression or the decrease of the executer of miRNA repression (i.e. Argonaut proteins). The miR294/302 family includes 6 members (miR-291a/294/295-3p and miR-302a/b/d-3p) with the same seed sequence. miRNA profiling data and qRT-PCR showed that the total level of miR-294/302 family was not significantly altered at low $\mathrm{pH}$ conditions (Supplementary Fig. 7c, d). We then checked the mRNA and protein level of AGO1 and $\mathrm{AGO} 2$, since $\mathrm{AGO} 3$ and $\mathrm{AGO} 4$ were not expressed in ESCs. Interestingly, while both protein and mRNA of Ago2 changed little, AGO1 protein but not Ago1 mRNA was significantly downregulated in $2 \mathrm{i}+\mathrm{LIF}$ ESCs upon low $\mathrm{pH}$ treatment (Fig. 6 f and Supplementary Fig. 7e, f). We further confirmed that AGO1 protein was also downregulated in conventional serum cultured ESCs (Fig. 6g). More interestingly, low $\mathrm{pH}$ treatment also led to significant reduction of AGO1 protein in various human cancer cell lines (Fig. 6h). The reduction of AGO1 is consistent with EMT promoting effects of low $\mathrm{pH}$, since miRNAs including miR-200, miR-29 and miR-34 families have been shown to suppress EMT in various cancer ${ }^{40}$. Indeed, we found that mRNA targets of these miRNAs are enriched in upregulated genes in low pH treated U2OS cells (Fig. 6i). Altogether, these data show that low $\mathrm{pH}$ downregulates AGO1 protein to de-repress a subset of miRNA targets in ESCs and cancer cells.

\section{Discussion}

Why PSCs prefer aerobic glycolysis over oxidative phosphorylation is not clear. While previous research has focused on identifying intermediate metabolites that are essential for the epigenetic regulation of pluripotency ${ }^{1,2,14,17}$, the function of microenvironment shaped by lactic acidosis is largely overlooked. Here we present evidence that acidic microenvironment due to lactate production helps maintain the self-renewal and 
pluripotency of mouse and human ESCs (Fig. 7). RNA-Seq, miRNA Seq and OCT4, H3K4me3 and H3K27me3 ChIP-Seq confirmed that low-pH treated ESCs are indeed pluripotent at transcriptomic and epigenomic levels. Mechanistically, we showed that low-pH treatment maintains ESC self-renewal and pluripotency through both epigenetic and post-transcriptional mechanisms. First, the tri-methylation of H3K27 is globally reduced at acidic conditions. Second, acidic $\mathrm{pH}$ stabilized hundreds of mRNAs including many pluripotency genes. In addition, we found that AGO1 protein is downregulated at acidic $\mathrm{pH}$, leading to the global upregulation of miR-290/302 targets in ESCs. Importantly, we found that AGO1 downregulation and mRNA stabilization by low-pH treatment are also conserved in cancer cells, indicating the conservation of a common pathway mediating low $\mathrm{pH}$ response in different cell lineages. Altogether, our study provides insights into mechanisms whereby acidic microenvironment shaped by enhanced glycolysis regulates gene expression and cell fate. In addition, our findings have broad implications for the fields of regenerative medicine and cancer.

The $\mathrm{pH}$ range 6.4-6.8 used in this study is physiologically relevant for mouse and human blastocysts as well as human tumors ${ }^{20,41}$. Acidosis is associated with multiple malignant phenotypes in tumors including increased metastasis and radioresistance ${ }^{21,}$ ${ }^{22}$. In contrast, the role of acidic environment in blastocysts remains elusive. Our in vitro study points to an interesting hypothesis that lactic acid may work together with other signaling pathways to maintain the self-renewal and pluripotency of epiblast cells during early embryonic stages. Consistent with this, embryos passing 8-cell stage, approximately when the metabolism of an embryo shifts to glycolysis, prefers culture media with lower $\mathrm{pH}$ for full development ${ }^{42}$. In addition, Ldha knockout leads to preimplantation death in mouse embryos (The Sanger Mouse Genetics Program). Future work should elucidate the functional roles of lactate accumulation in embryo development. These studies may lead to the development of better means to culture embryos for in vitro fertilization (IVF) practice. 
Enhanced glycolysis is observed in a variety of stem cells and cancer cells ${ }^{1-5}$. Elucidating its function and working mechanism can provide insights on how to manipulate stem cells and cure cancers. However, directly manipulating $\mathrm{pH}$ in vivo to change the microenvironment of stem cells and cancers is apparently not feasible. Therefore, understanding the downstream molecular impact of extracellular acidity is of high importance, as this will identify candidate pathways that may be manipulated to control stem cells or cancer growth. Our study for the first time makes connections between the extracellular acidity and post-transcriptional regulation in ESCs and cancer cells. RNA binding proteins, miRNAs as well as mRNA modifications (e.g. m6A) are major post-transcriptional regulators that have demonstrated functions in stem cells and cancer cells ${ }^{43-45}$. Future studies are warranted to elucidate the components of these pathways and other novel pathways that link extracellular acidity to post-transcriptional regulation. Studying these components may lead to the invention of innovative approaches in regenerative medicine and cancer therapy. 


\section{Materials and Methods}

\section{Cell culture and differentiation assay}

Mouse ESCs were cultured on $0.1 \%$ gelatin-coated plates in $2 \mathrm{i}+\mathrm{LIF}$ media which is consist of N2B27 supplemented with PD0325901 (1.0 $\mu \mathrm{M})$, CHIR99021 $(3.0 \mu \mathrm{M})$ and leukemia inhibitory factor (LIF, 1000 unit /ml) or serum media which is consist of DMEM supplemented with 15\% FBS and LIF. For differentiation assay, ESCs were first plated in $2 \mathrm{i}+\mathrm{LIF}$ for $20 \mathrm{hr}$, then washed once with 1xPBS and changed to N2B27 with indicated supplements, cultured for another 2 days before analysis with media changed every day; For colony formation assay, 200 cells were plated on a well of 12well on $0.1 \%$ gelatin-coated plate in $2 \mathrm{i}+\mathrm{LIF}$ media. Human ESCs were cultured on MEF in KSR medium knockout DMEM with $20 \%$ KSR supplemented with $100 \mathrm{mM} 2$ mercaptoethanol (2ME), MEM nonessential amino acids (NEAA), 2 mM L-glutamine and 5ng/ml bFGF (Leto Laboratories, Beijing). For differentiation assay, 300 single hESCs were plated on MEF in KSR medium with bFGF adding Y27632(10uM), then bFGF and Y27632 were withdrawn second day and changed medium every two days until the clone is formulated. Immediately before media change, $\mathrm{pH}$ of media was adjusted by lactic acid, hydrochloride acid and sulphuric acid and measured by $\mathrm{pH}$ meter at room temperature.

\section{RNA extraction and qRT-PCR}

Total RNA was extracted following standard Trizol protocol (Invitrogen). RT-PCR was performed using Sybr Green mix (Vazyme) with ABI Step One Plus (Applied Biosystems).

\section{RNA-seq, Small RNA-seq and bioinformatics analysis}

RNA and small RNA libraries from two independent biological replicates were generated using NEBNext small RNA library Prep Set for Illumina (E7420) and NEBNext Small RNA Library Prep Set (E7330S) according to the manufacturer's instruction, respectively. NEBNext multiplex oligos for Illumina (E7335) were used. The final libraries were measured by Qubit fluorometric assay (Life Technologies). 
Libraries were sequenced by Illumina HiSeq 2500. Data was analyzed as previously described $^{39}$. R 3.1.1 was used for generation of the heatmap and PCA plot. List of selected genes related to pluripotency and differentiation is as previously published ${ }^{46}$. GSEA2.4 was used to test for the enrichment of selected gene sets by java GSEA Desktop Application.

\section{ChIP-qPCR and ChIP-seq}

Cells under different culture conditions were cross-linked with $1 \%$ formaldehyde for $15 \mathrm{~min}$ at room temperature followed by the addition of $125 \mathrm{mM}$ glycine to inactivate formaldehyde. Chromatin extracts containing DNA fragments with an average size of 200-500 bps were immunoprecipitated using IgG or antibodies against H3K27me3 (Abclonal, A2363), H3K4 me3 (Abclonal, A2357) and OCT4 (Santa, sc-8628) at $4^{\circ} \mathrm{C}$ overnight. ChIP-Seq library was generated by NEBNext Ultra DNA Library Prep Kit (E7370). Libraries were submitted for sequencing on Illumina Hiseq 2000. Sequenced reads after trimmed were mapped to mouse mm10 whole genome using STAR v2.3.0. Peaks were called by MACS2 with default parameters ( $p$ value $\leqslant 0.01$ and --SPMR). Intensity plot and heatmap were generated by ngs.plot.r with aligned BAM files. Peak plot was generated by IGV software.

\section{mRNA half-life analysis}

To measure mRNA stability, transcription was blocked by adding actinomycin D (Sigma, cat \# A-9415) to the medium at the concentration of $10 \mathrm{mg} / \mathrm{ml}$. Cells were harvested at $0,2,4$ and $8 \mathrm{~h}$ after the addition of actinomycin $\mathrm{D}$ and processed for RNAseq or qRT-PCR preparation and analysis. For RNA half-life calculation, genes with average FPKM lower than 0.2 in all samples were deemed as lowly expressed and were removed from further analysis. Filtered genes were normalized to $\beta$-actin. Then a linear regression of the form $y=a-b t$ was performed on each gene through the four time points, where $\mathrm{y}$ is the log-transformed (base 10) read count, $\mathrm{t}$ is the time, $\mathrm{b}$ is the slope, $\mathrm{a}$ is the intercept, and $\mathrm{d}=\mathrm{b} \times \ln (10)$ is the instantaneous decay rate. Half-life of each gene was calculated and projected as $\mathrm{H}=\min (24, \ln (2) / \mathrm{d})$ for positive $\mathrm{d}$ and $\mathrm{h}=24 \mathrm{hr}$ 
for negative d. Genes that taken for further analysis were determined by requiring correlation $(\mathrm{R})$ of linear data was $<-0.7$ in all samples.

\section{miR-294/302 target analysis}

A gene is defined as a miR-294/302 target in ESCs based on two criteria: First, it has at least one predicted miRNA binding sites complementary to the seed sequence "AAGUGC"; Second, its expression is significantly upregulated in Dgcr8 knockout versus wild type ESCs (top 10\% ranked by fold changes, > 1.99 fold). Similarly, top $10 \%$ genes with increased mRNA half-lives (> 1.83 fold) were used to make Venn diagram in Figure 6.

\section{Western blot analysis}

Antibodies against NANOG from Calbiochem (Darmstadt, Germany), ACTIN (AP0060) and TUBBLIN (AP0064) was from Bioworld Technology (Nanjing, China). Anti-rabbit and mouse secondary antibodies was from LI-COR and membranes were imaged using Oddssay.

\section{Statistical analysis}

The data were presented as mean \pm SD except where indicated otherwise. We performed two-tailed unpaired Student's t-test to determine statistical significance except for analysis shown in the boxplot graph (two-tailed Wilcoxon signed-rank test), cumulative plot (Kolmogorov-Smirnov test) and Venn diagram (chi-square test). P value $<0.05$ was considered as statistically significant.

\section{Acknowledgements}

We would like to thank members of Wang laboratory for critical reading and discussion of the manuscript. This study was supported by The National Key Research and Development Program of China [2016YFA0100701] and the National Natural Science Foundation of China [31471222, 31622033 and 31521062] to YW. 


\section{Author Contributions}

WTG performed most of experiments with help from other authors. SHW performed experiments in Figure 3. XSZ and XWW performed experiments related Eed knockout. KLG and JH helped with bioinformatics analysis. All authors were involved in the interpretation of data. YW and WTG conceived the project. YW supervised the project. YW and WTG wrote the manuscript with help from other authors.

\section{Availability of Data and Materials}

All data generated or analysed during this study are included in the manuscript and its supplementary information files. Original sequencing data used and/or analysed during the current study are available from the corresponding author on reasonable request. 


\section{References}

1. Vander Heiden, M.G., Cantley, L.C. \& Thompson, C.B. Understanding the Warburg effect: the metabolic requirements of cell proliferation. Science 324, 1029-1033 (2009).

2. Lunt, S.Y. \& Vander Heiden, M.G. Aerobic glycolysis: meeting the metabolic requirements of cell proliferation. Annu Rev Cell Dev Biol 27, 441-464 (2011).

3. Cliff, T.S. \& Dalton, S. Metabolic switching and cell fate decisions: implications for pluripotency, reprogramming and development. Current opinion in genetics \& development 46, 44-49 (2017).

4. Shyh-Chang, N. \& Daley, G.Q. Metabolic switches linked to pluripotency and embryonic stem cell differentiation. Cell metabolism 21, 349-350 (2015).

5. Ito, K. \& Suda, T. Metabolic requirements for the maintenance of self-renewing stem cells. Nature reviews. Molecular cell biology 15, 243-256 (2014).

6. Folmes, C.D., Dzeja, P.P., Nelson, T.J. \& Terzic, A. Metabolic plasticity in stem cell homeostasis and differentiation. Cell stem cell 11, 596-606 (2012).

7. Teslaa, T. \& Teitell, M.A. Pluripotent stem cell energy metabolism: an update. The EMBO journal 34, 138-153 (2015).

8. Cliff, T.S. et al. MYC Controls Human Pluripotent Stem Cell Fate Decisions through Regulation of Metabolic Flux. Cell stem cell 21, $502-516$ e509 (2017).

9. Gu, W. et al. Glycolytic Metabolism Plays a Functional Role in Regulating Human Pluripotent Stem Cell State. Cell stem cell 19, 476-490 (2016).

10. Sone, M. et al. Hybrid Cellular Metabolism Coordinated by Zic3 and Esrrb Synergistically Enhances Induction of Naive Pluripotency. Cell metabolism 25, 1103-1117 e1106 (2017).

11. Cao, Y. et al. miR-290/371-Mbd2-Myc circuit regulates glycolytic metabolism to promote pluripotency. The EMBO journal 34, 609-623 (2015).

12. Cha, Y. et al. Metabolic control of primed human pluripotent stem cell fate and function by the miR-200c-SIRT2 axis. Nature cell biology 19, 445-456 (2017).

13. Zhou, W. et al. HIF1alpha induced switch from bivalent to exclusively glycolytic metabolism during ESC-to-EpiSC/hESC transition. The EMBO journal 31, 21032116 (2012).

14. Moussaieff, A. et al. Glycolysis-Mediated Changes in Acetyl-CoA and Histone Acetylation Control the Early Differentiation of Embryonic Stem Cells. Cell metabolism 21, 392-402 (2015).

15. Atlasi, Y. \& Stunnenberg, H.G. The interplay of epigenetic marks during stem cell differentiation and development. Nature reviews. Genetics 18, 643-658 (2017).

16. Boland, M.J., Nazor, K.L. \& Loring, J.F. Epigenetic Regulation of Pluripotency and Differentiation. Circulation Research 115, 311-324 (2014).

17. Carey, B.W., Finley, L.W., Cross, J.R., Allis, C.D. \& Thompson, C.B. Intracellular alpha-ketoglutarate maintains the pluripotency of embryonic stem cells. Nature 518, 413-416 (2015).

18. Gardner, D.K. \& Harvey, A.J. Blastocyst metabolism. Reproduction, fertility, and development 27, 638-654 (2015). 
19. Lees, J.G., Gardner, D.K. \& Harvey, A.J. Pluripotent Stem Cell Metabolism and Mitochondria: Beyond ATP. Stem Cells Int 2017, 2874283 (2017).

20. Gardner, D.K. Lactate production by the mammalian blastocyst: manipulating the microenvironment for uterine implantation and invasion? BioEssays : news and reviews in molecular, cellular and developmental biology 37, 364-371 (2015).

21. Swietach, P., Vaughan-Jones, R.D., Harris, A.L. \& Hulikova, A. The chemistry, physiology and pathology of $\mathrm{pH}$ in cancer. Philosophical transactions of the Royal Society of London. Series B, Biological sciences 369, 20130099 (2014).

22. Damaghi, M., Wojtkowiak, J.W. \& Gillies, R.J. pH sensing and regulation in cancer. Front Physiol 4, 370 (2013).

23. Rofstad, E.K., Mathiesen, B., Kindem, K. \& Galappathi, K. Acidic extracellular $\mathrm{pH}$ promotes experimental metastasis of human melanoma cells in athymic nude mice. Cancer Res 66, 6699-6707 (2006).

24. Ulmschneider, B. et al. Increased intracellular $\mathrm{pH}$ is necessary for adult epithelial and embryonic stem cell differentiation. The Journal of cell biology 215, 345-355 (2016).

25. Wray, J. et al. Inhibition of glycogen synthase kinase-3 alleviates Tcf3 repression of the pluripotency network and increases embryonic stem cell resistance to differentiation. Nature cell biology 13, 838-845 (2011).

26. Betschinger, J. et al. Exit from pluripotency is gated by intracellular redistribution of the bHLH transcription factor Tfe3. Cell 153, 335-347 (2013).

27. Ng, H.H. \& Surani, M.A. The transcriptional and signalling networks of pluripotency. Nature cell biology 13, 490-496 (2011).

28. Juan, Aster H. et al. Roles of H3K27me2 and H3K27me3 Examined during Fate Specification of Embryonic Stem Cells. Cell Reports 17, 1369-1382 (2016).

29. Chen, Q. \& Hu, G. Post-transcriptional regulation of the pluripotent state. Current opinion in genetics \& development 46, 15-23 (2017).

30. Lou, C.H., Shum, E.Y. \& Wilkinson, M.F. RNA degradation drives stem cell differentiation. The EMBO journal 34, 1606-1608 (2015).

31. Zhao, B.S. \& He, C. Fate by RNA methylation: m6A steers stem cell pluripotency. Genome biology 16, 43 (2015).

32. Hao, J., Duan, F.F. \& Wang, Y. MicroRNAs and RNA binding protein regulators of microRNAs in the control of pluripotency and reprogramming. Current opinion in genetics \& development 46, 95-103 (2017).

33. Percharde, M. et al. Ncoa3 functions as an essential Esrrb coactivator to sustain embryonic stem cell self-renewal and reprogramming. Genes \& development $\mathbf{2 6}$, 2286-2298 (2012).

34. Niwa, H., Burdon, T., Chambers, I. \& Smith, A. Self-renewal of pluripotent embryonic stem cells is mediated via activation of STAT3. Genes \& development 12, 2048-2060 (1998).

35. Aksoy, I. et al. Self-renewal of murine embryonic stem cells is supported by the serine/threonine kinases Pim-1 and Pim-3. Stem cells 25, 2996-3004 (2007).

36. Martinez-Zaguilan, R. et al. Acidic $\mathrm{pH}$ enhances the invasive behavior of human melanoma cells. Clin Exp Metastasis 14, 176-186 (1996). 
37. Suzuki, A., Maeda, T., Baba, Y., Shimamura, K. \& Kato, Y. Acidic extracellular $\mathrm{pH}$ promotes epithelial mesenchymal transition in Lewis lung carcinoma model. Cancer cell international 14, 129 (2014).

38. Wang, X.W. et al. A DGCR8-Independent Stable MicroRNA Expression Strategy Reveals Important Functions of miR-290 and miR-183-182 Families in Mouse Embryonic Stem Cells. Stem cell reports (2017).

39. Gu, K.L. et al. Pluripotency-associated miR-290/302 family of microRNAs promote the dismantling of naive pluripotency. Cell research 26, 350-366 (2016).

40. Nicoloso, M.S., Spizzo, R., Shimizu, M., Rossi, S. \& Calin, G.A. MicroRNAs--the micro steering wheel of tumour metastases. Nature reviews. Cancer 9, 293-302 (2009).

41. Hashim, A.I., Zhang, X., Wojtkowiak, J.W., Martinez, G.V. \& Gillies, R.J. Imaging $\mathrm{pH}$ and metastasis. NMR in biomedicine 24, 582-591 (2011).

42. Hentemann, M., Mousavi, K. \& Bertheussen, K. Differential pH in embryo culture. Fertil Steril 95, 1291-1294 (2011).

43. Zhao, B.S., Roundtree, I.A. \& He, C. Post-transcriptional gene regulation by mRNA modifications. Nature reviews. Molecular cell biology 18, 31-42 (2017).

44. Schoenberg, D.R. \& Maquat, L.E. Regulation of cytoplasmic mRNA decay. Nature reviews. Genetics 13, 246-259 (2012).

45. Fabian, M.R., Sonenberg, N. \& Filipowicz, W. Regulation of mRNA translation and stability by microRNAs. Annual review of biochemistry 79, 351-379 (2010).

46. Kumar, R.M. et al. Deconstructing transcriptional heterogeneity in pluripotent stem cells. Nature 516, 56-61 (2014). 


\section{FIGURE LEGENDS}

Figure 1. Lactic acid but not lactate promotes the self-renewal and pluripotency of mouse ESCs. (a-c) FACS analysis of GFP positive portions of Rex1-d2EGFP ESCs under different culture conditions; $10 \mathrm{mM}$ lactic acid or lactate was used. (d) qRT-PCR analysis of pluripotency markers in low pH treated mouse ESCs; The Rpl7 gene was used as a control. Data were normalized to the mRNA level of $2 \mathrm{i}+\mathrm{LIF}$ cultured mouse ESCs. Shown are mean $\pm \mathrm{SD}, \mathrm{n}=3$. (e) Western analysis of NANOG protein levels. (f) Morphology of low-pH treated mESC colonies. (g, h) Colony formation analysis. Representative images for whole well are shown for alkaline phosphatase (AP) staining. Colony numbers are shown as mean $\pm \mathrm{SD}, \mathrm{n}=3$. (i) qRT-PCR analysis of pluripotency markers in ESCs cultured in serum media with $1 \mu \mathrm{M}$ retinoid acid and with or without HEPES buffer; The Rpl7 gene was used as a control. Data were normalized to the mRNA level of $2 \mathrm{i}+$ LIF cultured mouse ESCs. Shown are mean $\pm \mathrm{SD}, \mathrm{n}=3$.

Figure 2. Transcriptomic and epigenomic analyses confirm that acidic condition maintains the pluripotency of ESCs. (a) PCA analysis for selected genes that are related to pluripotency and differentiation. (b) PCA analysis of global miRNA expression. (c, d) Heatmap and signal intensity plot of OCT4 ChIP. Shown are $\pm 1.5 \mathrm{~kb}$ regions around the peak center of all peaks called by MACS2 (p value < 0.01); (e, f) Heatmap and signal intensity plot of H3K27me3 ChIP. Shown are promoters containing H3K27me3 peaks called by MACS2 ( $\mathrm{p}$ value < 0.01 ) over random distribution in at least one of the culture conditions. (g, h) Heatmap and signal intensity plot of H3K4me3 ChIP. Shown are promoters containing $\mathrm{H} 3 \mathrm{~K} 4 \mathrm{me} 3$ peaks called by MACS2 (p value < 0.01 ) over random distribution in at least one of the culture conditions.

Figure 3. Low pH promotes the self-renewal and pluripotency of human ESCs. (a) Morphology of low-pH treated human ESCs. (b) qRT-PCR analysis of pluripotency markers of low-pH treated human ESCs. The $\beta$-actin gene was used as a control. Data were normalized to the mRNA level of bFGF cultured human ESCs. Shown are mean 
$\pm \mathrm{SD}, \mathrm{n}=3$. (c, d) FACS analysis of GFP positive portions of OCT4-GFP reporter human ESCs under different culture conditions.

Figure 4. Low pH causes global reduction of H3K27me3 at transcriptional start sites. (a) Box plot for $\log 2$ fold change of mRNA expression for all expressed genes and genes that have H3K27 me3 peaks in 2i+LIF cultured mESCs. (b) Heatmap of H3K27me3 ChIP signals for promoters gained H3K27me3 signals during differentiation. (c) Genome browser screenshot of the H3K27me3 ChIP reads aligning to Hoxa locus. (d) Venn diagrams showing the overlaps between genes upregulated in Eed knockout mESCs and in pH6.4 treated mESCs. (e) GSEA analysis for $>2$ fold upregulated genes in Eed knockout mouse ESCs between pH7.4 and pH6.4 2i+LIF culture conditions. (f) qRT-PCR analysis of pluripotency markers in wild-type mESCs and Eed KO mouse ESCs differentiated for 1 day in N2B27 media without $2 \mathrm{i}$ and LIF. The $\beta$-actin gene was used as a control. Data were normalized to the mRNA level of $2 \mathrm{i}+\mathrm{LIF}$ cultured mESCs. Shown are mean $\pm \mathrm{SD}, \mathrm{n}=2$.

Figure 5. Low pH stabilizes thousands of mRNAs including known pluripotency genes. (a) mRNA degradation plot of representative genes. The $\beta$-actin gene was used as a control. For each gene, data were normalized to ESCs cultured in each condition without actinomycin D treatment (0 hour). Shown are mean $\pm S D, n=2$. Actinomycin $D$ was added $48 \mathrm{hr}$ after low $\mathrm{pH}$ treatment, and cells were collected at $0,2,4$, and $8 \mathrm{hr}$ after actinomycin D addition for RNA-seq. (b) Cumulative plot for mRNA half-life of ESCs in pH7.4 and pH6.4 culture conditions. (c) Bar plots of log2 fold change of mRNA halflife between pH6.4 and pH7.4. (d) Half-life of represented pluripotency genes Dppa5a and Fbxo15. The $\beta$-actin gene was used as a control. For each gene, data were normalized to ESCs cultured in each condition without actinomycin D treatment (0 hour). Shown are mean $\pm S D, n=2$. (e) Box plot for mRNA half-life of pluripotency genes in pH7.4 and pH6.4 culture conditions. (f) mRNA half-life analysis of pluripotency genes in ESCs after cultured in N2B27 media for 3 hours at pH 7.4 and pH 6.4. Data were normalized to mRNA half-life of each gene in $2 \mathrm{i}+\mathrm{LIF}$ ESCs. (g) 
Cumulative plot for mRNA half-life of U2OS cells in pH7.4 and pH6.4 culture conditions. (h) GSEA analysis for ECM gene set of U2OS cells in pH7.4 and pH6.4 culture conditions.

Figure 6. Low pH downregulates AGO1 protein expression and derepresses a subset of miRNA targets. (a) Venn diagrams showing the overlaps between genes with extended mRNA half-lives in pH6.4 condition and miR-294 target genes. (b) GSEA analysis for miR-294 target genes in pH7.4 and pH6.4 culture conditions. (c) qRT-PCR analysis of canonical miR-294 target genes (Fndc3b, Mbd2 and Tgfbr2) and Myc expression in low-pH treated mESCs; The Rpl7 gene was used as a control. Data were normalized to the mRNA level of $2 \mathrm{i}+\mathrm{LIF}$ cultured mouse ESCs. Shown are mean $\pm \mathrm{SD}, \mathrm{n}=3$. (d) Luciferase reporter assay. Shown are mean $\pm \mathrm{SD}, \mathrm{n}=3$. (e) qRT-PCR analysis of pluripotency markers in wild-type ESCs and Dgcr8 knockout mouse ESCs cultured for 2 days in N2B27 differentiation media. The $\beta$-actin gene was used as a control. Data were normalized to the mRNA level of $2 \mathrm{i}+\mathrm{LIF}$ cultured mouse ESCs. Shown are mean $\pm \mathrm{SD}, \mathrm{n}=2 ;(\mathbf{f}, \mathbf{g})$ Western analysis AGO1 in mESCs cultured in different $\mathrm{pH}$ conditions. The ACTIN or TUBBLIN was used as a loading control. (h) Western analysis AGO1 in human cancer cell lines U2OS and HepG2 in different $\mathrm{pH}$ condition. The TUBBLIN was used as a loading control. (i) GSEA of miRNA targets for genes upregulated ( 2 fold) in low $\mathrm{pH}$ treated U2OS cancer cells.

Figure 7. Summary graph showing that lactic acid generated from glycolysis promotes the self-renewal and pluripotency of ESCs. 
Figure 1

a

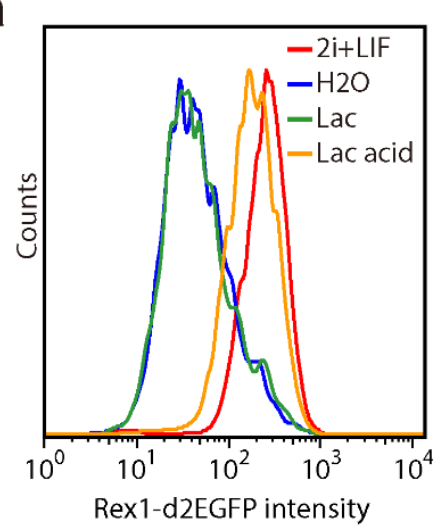

d

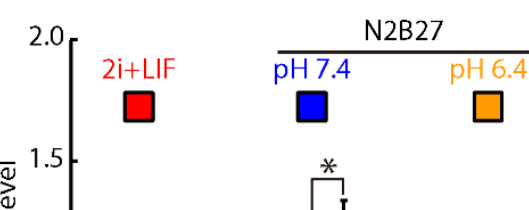

b

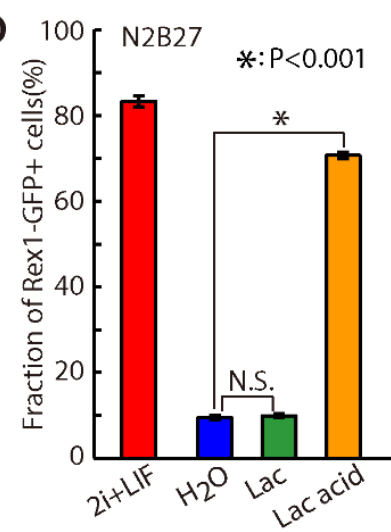

C

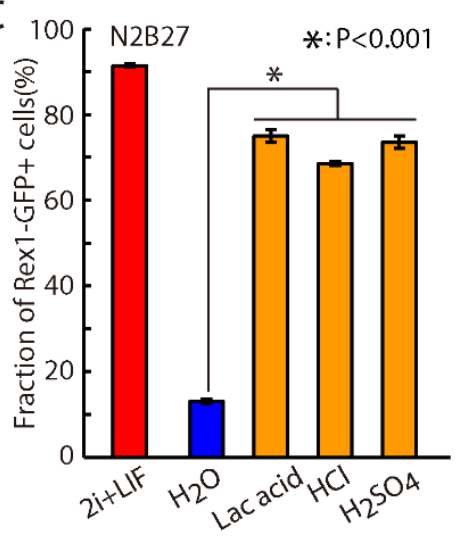

e

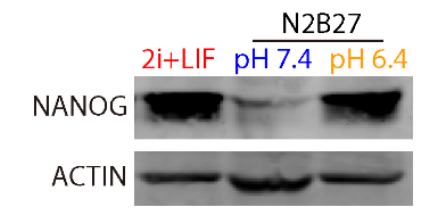

$\mathrm{pH} 7.4$

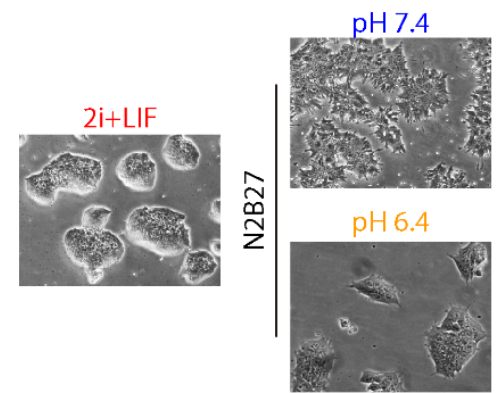

g
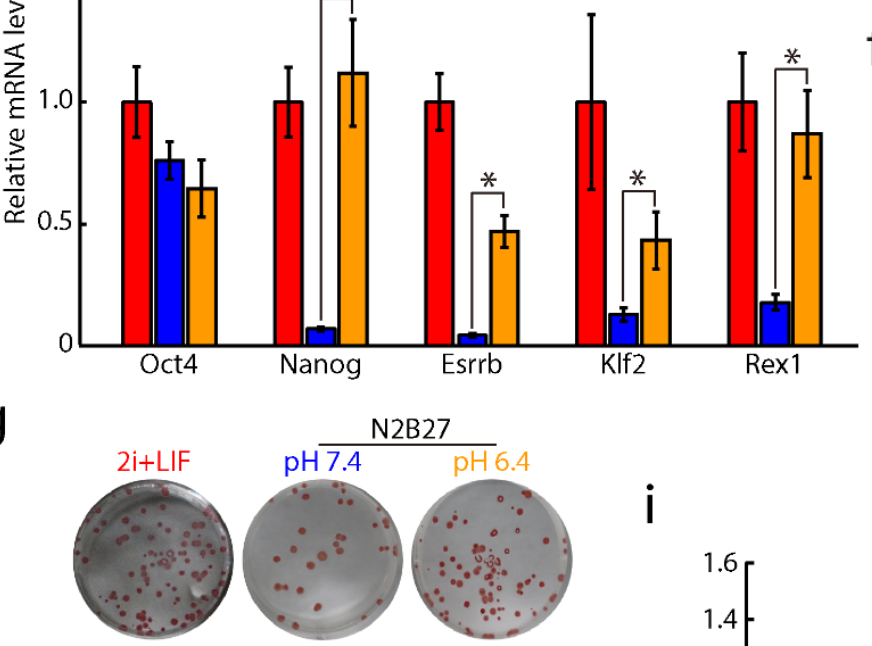

h

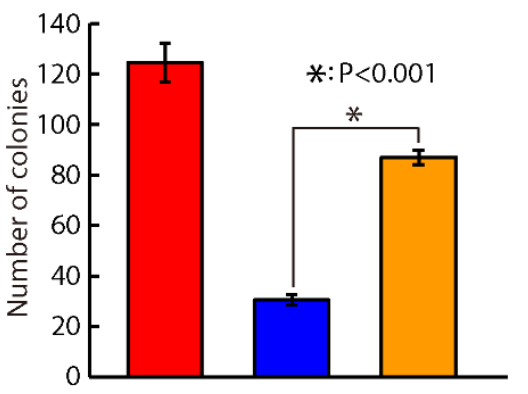

i Serum -LIF+RA

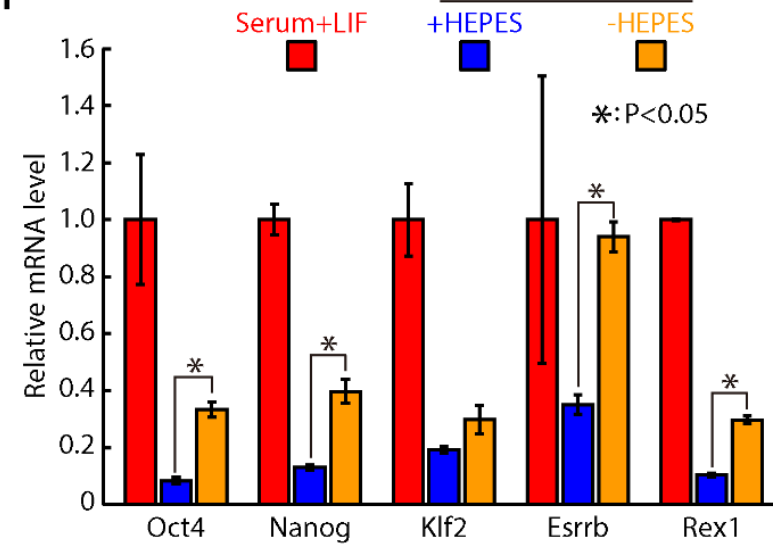


Figure 2

a

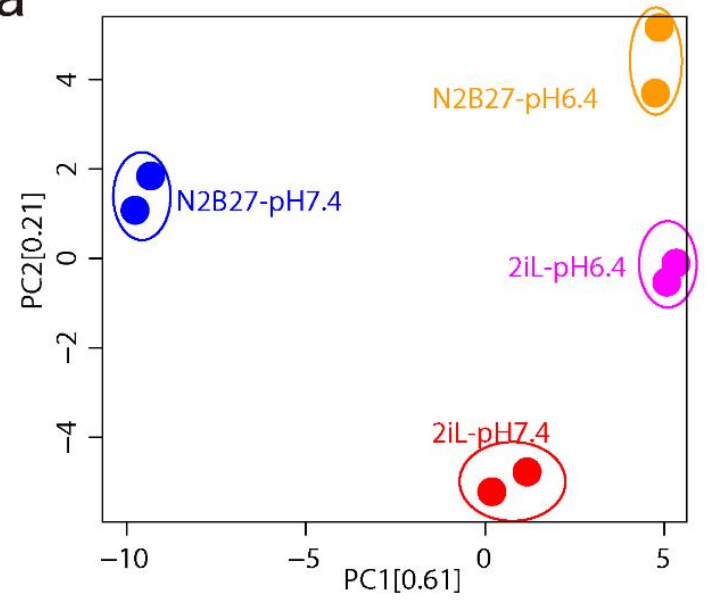

C

$\mathrm{pH} \frac{2 \mathrm{i}+\mathrm{LIF}}{7.4 \quad 6.4} \quad \frac{\mathrm{N} 2 \mathrm{~B} 27}{7.4 \quad 6.4}$

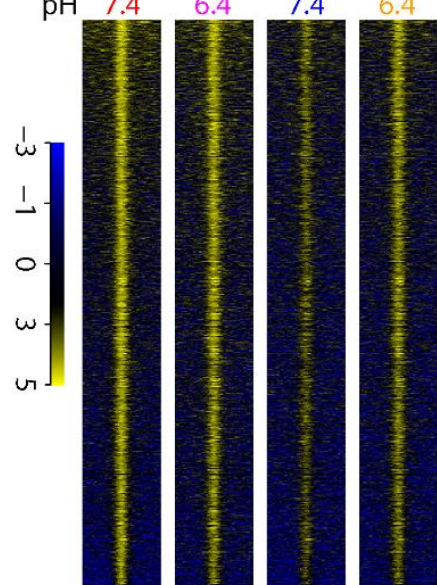

d

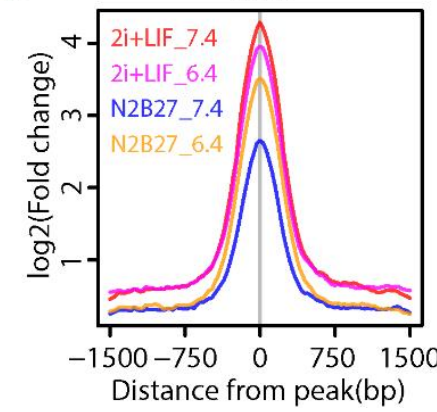

e

$f$ b
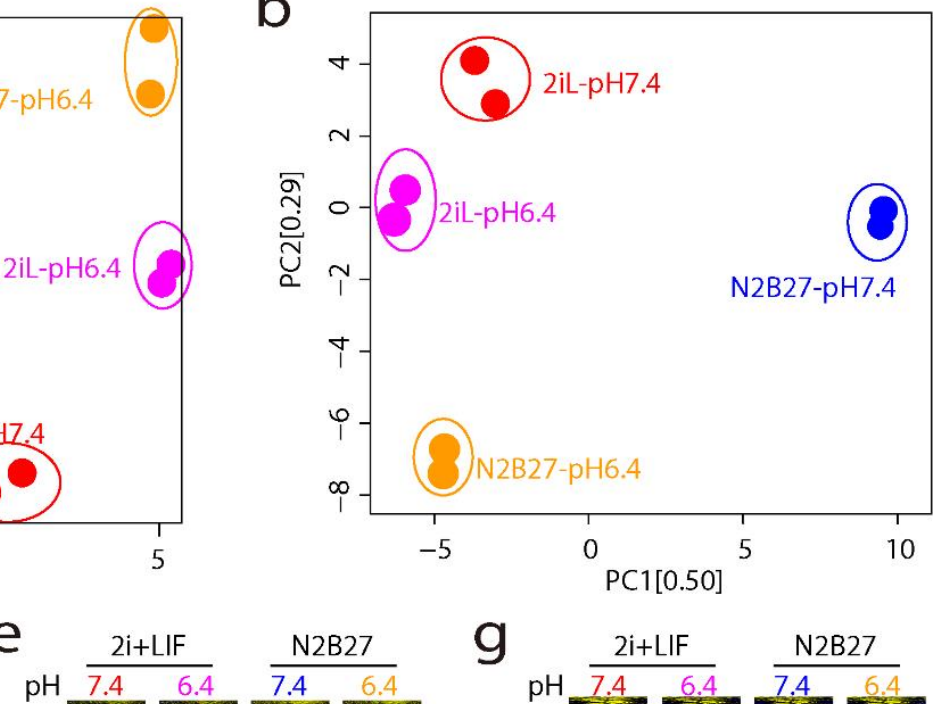

$\frac{\mathrm{N} 2 \mathrm{~B} 27}{7.4 \quad 6.4}$

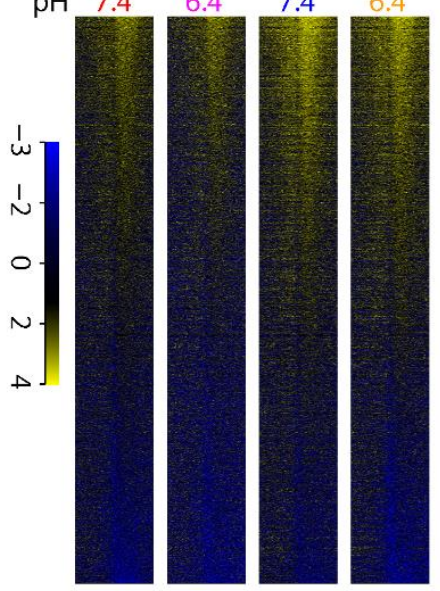

H3K27me3

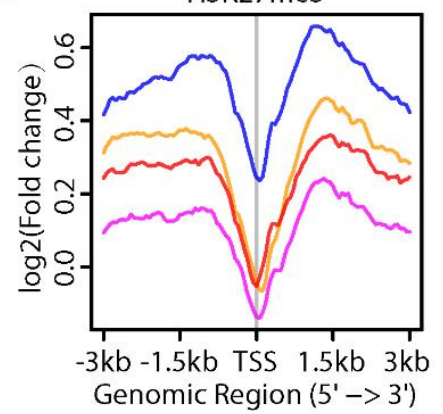

g

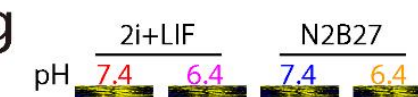

h
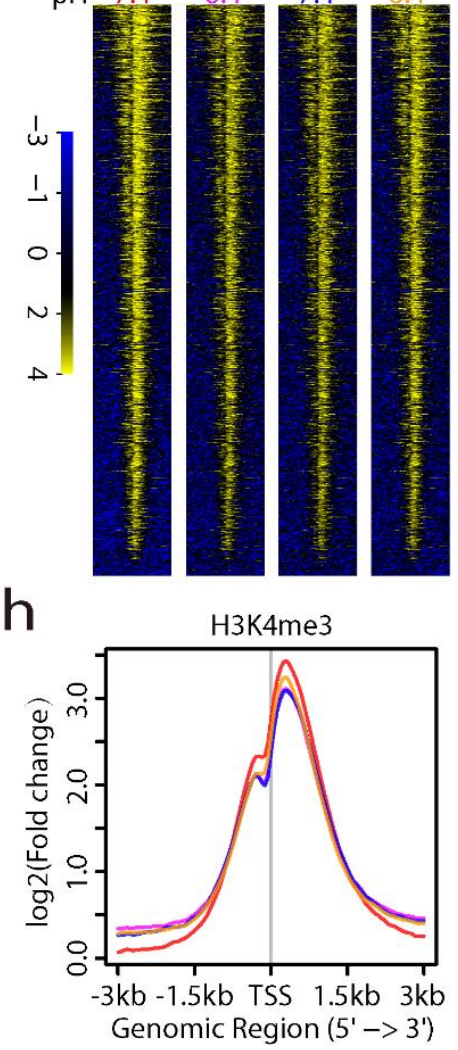


\section{Figure 3}

a

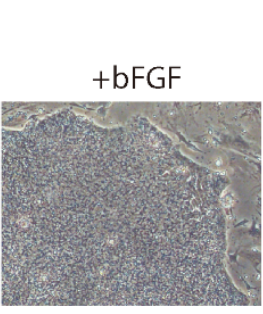

$\mathrm{pH} 7.5$

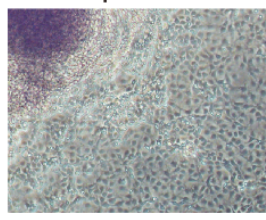

$-b F G F$

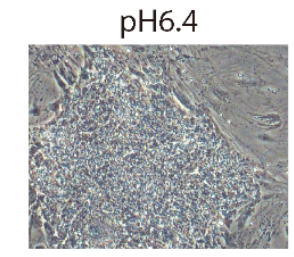

C

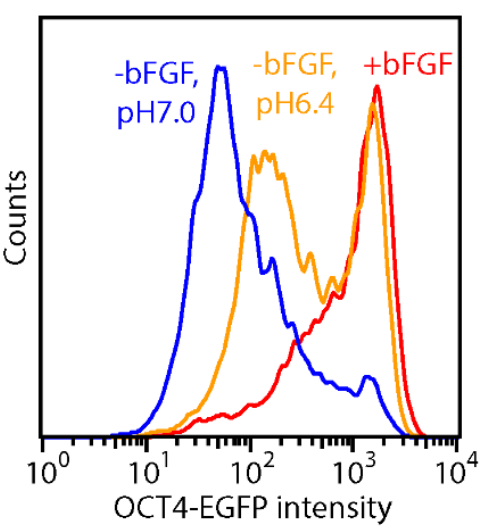

b

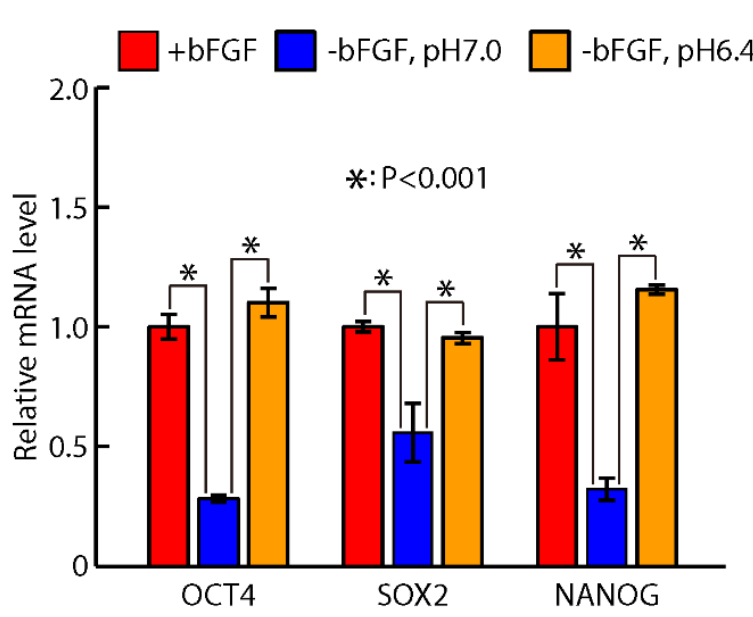

d

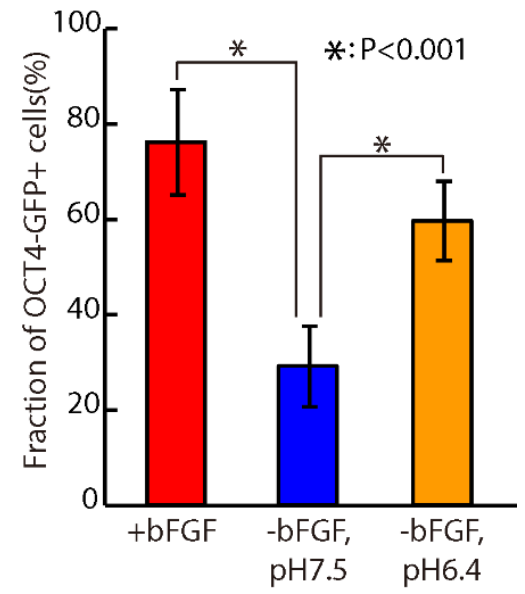




\section{Figure 4}

a

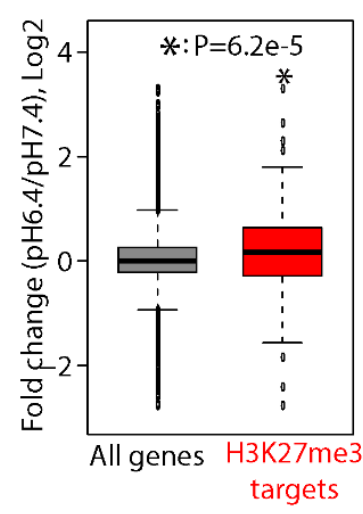

b

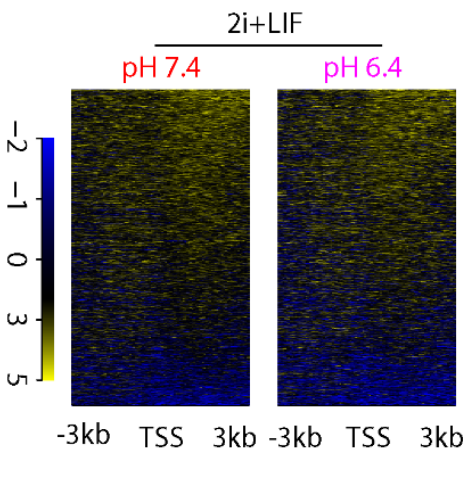

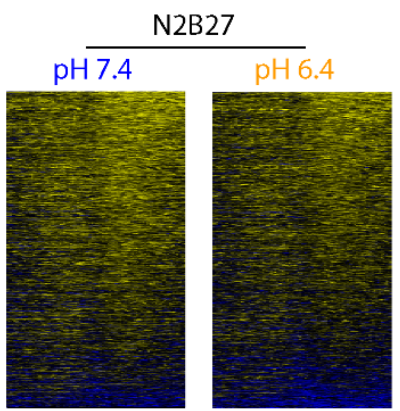

$-3 k b$ TSS $3 k b-3 k b$ TSS $3 k b$

C $5^{-}$

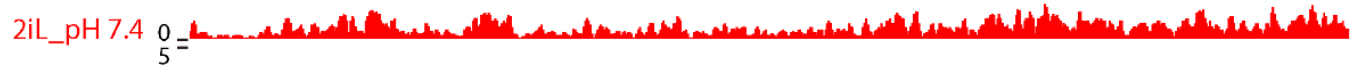

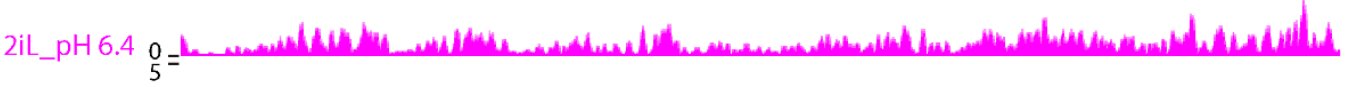

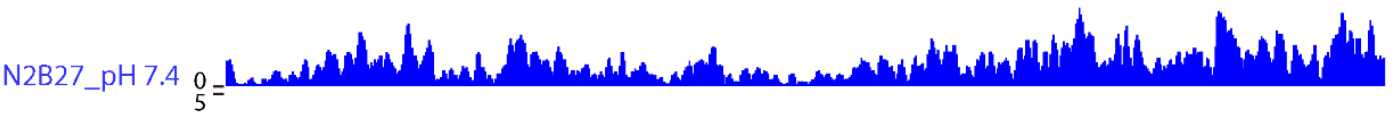
N2B27_pH 6.4 Hoxal 0 Hoxa4

d

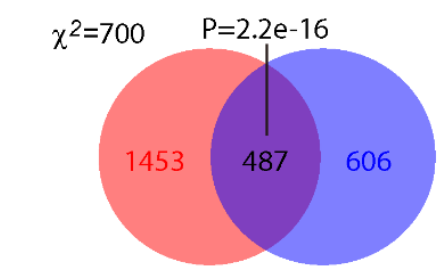

Eed KO_up2fold

pH 6.4_up2fold

$f$

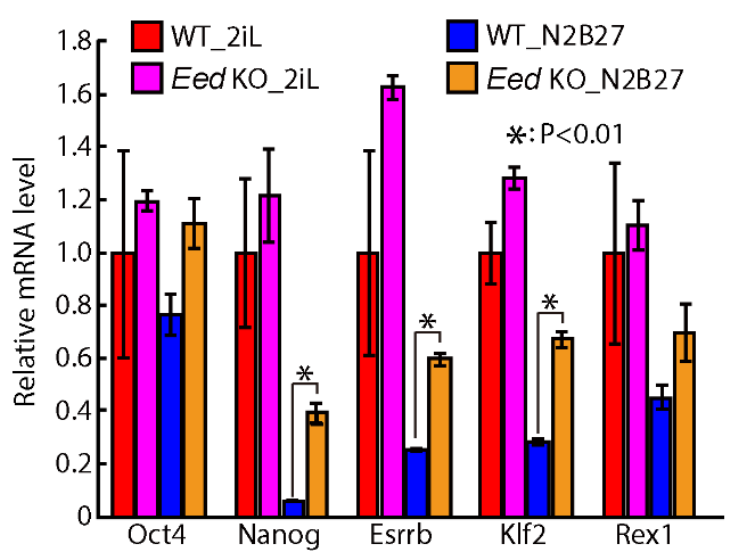

e

Gene Set Enrichment Analysis (GSEA) Eed KO_up 2 fold genes in pH6.4 treatment

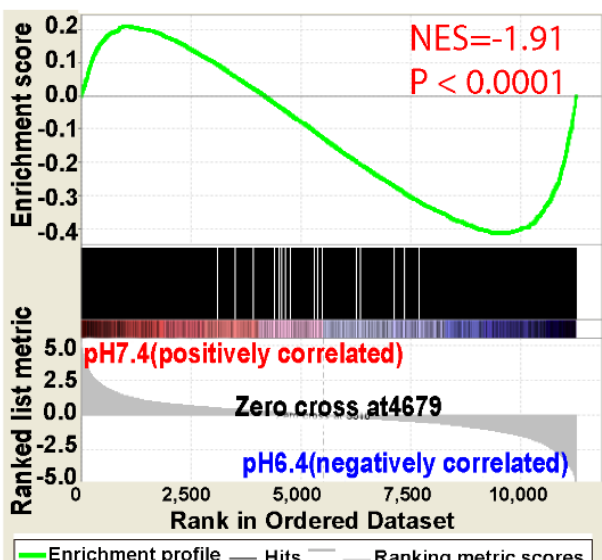




\section{Figure 5}
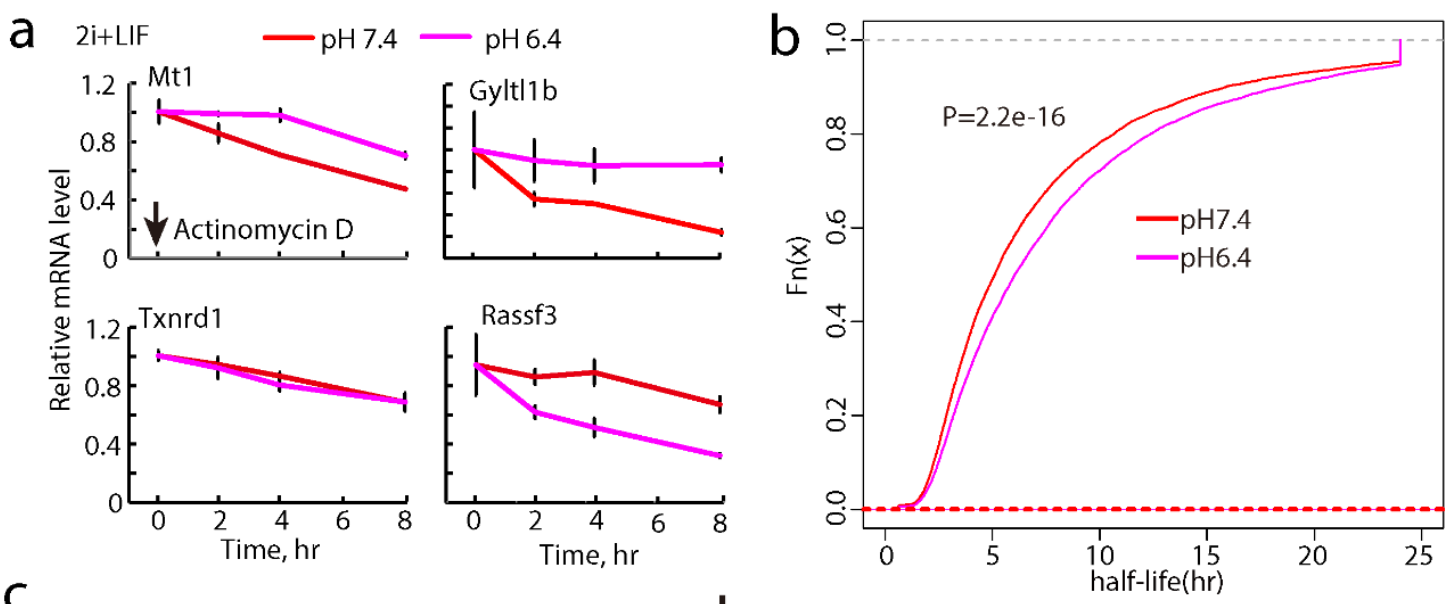

C

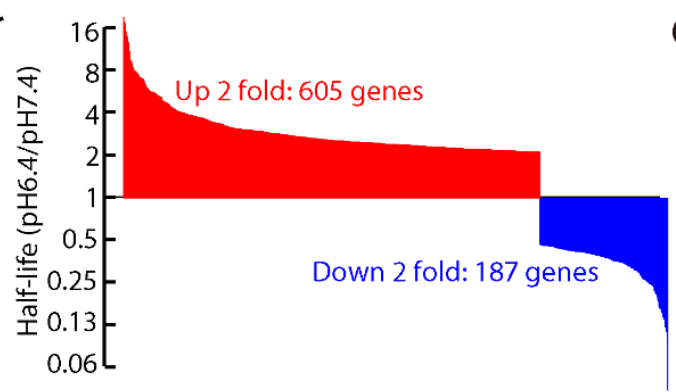

e

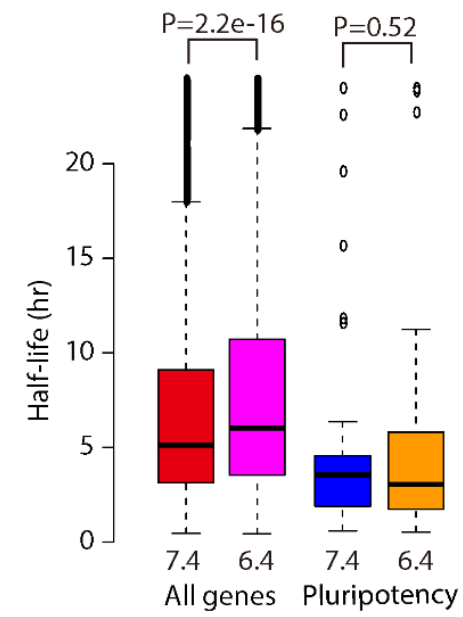

9

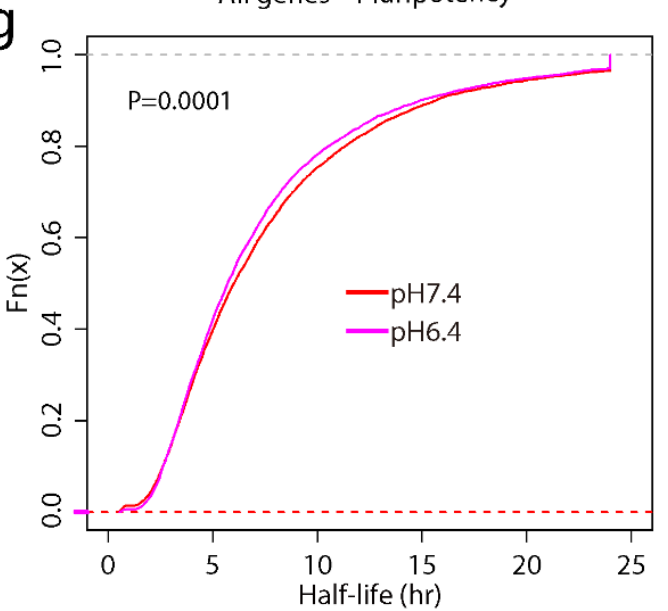

d

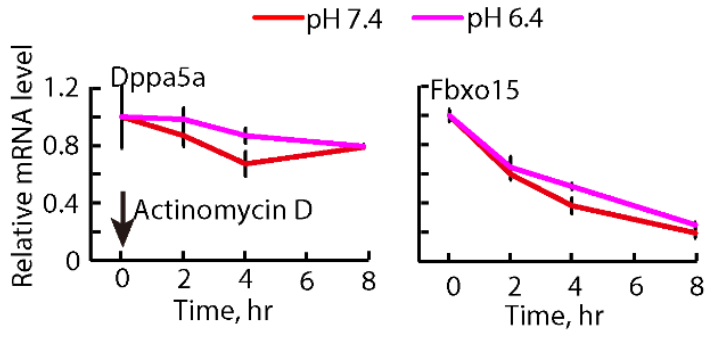

f

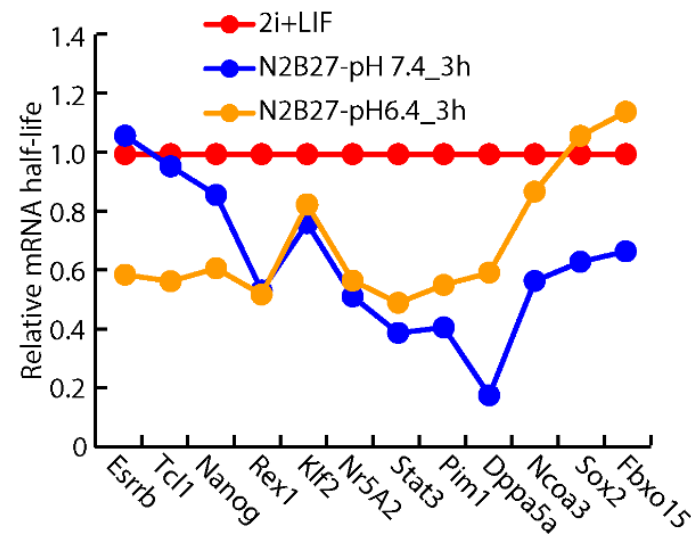

$\mathrm{h}$

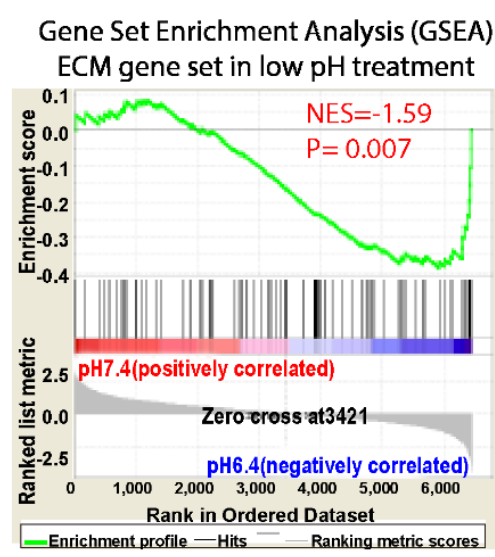




\section{Figure 6}

a

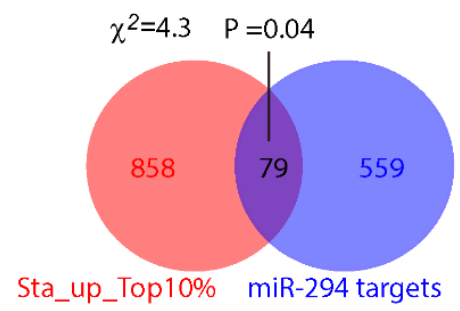

b Gene Set Enrichment Analysis (GSEA) miR-294 targets in $\mathrm{pH} 6.4$ treatment

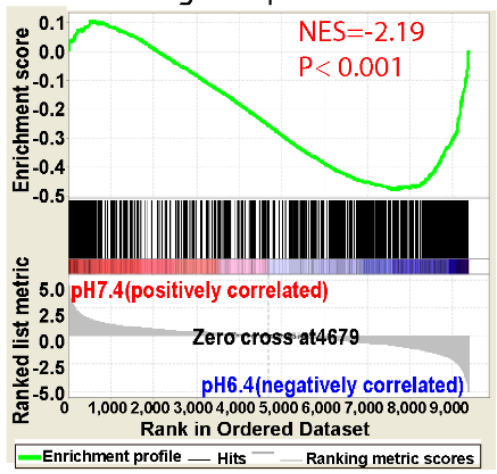

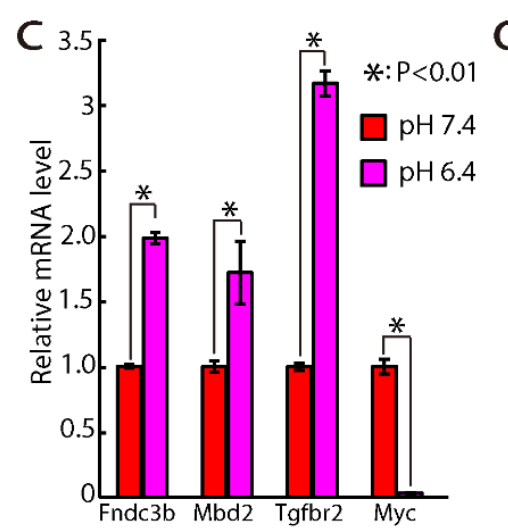

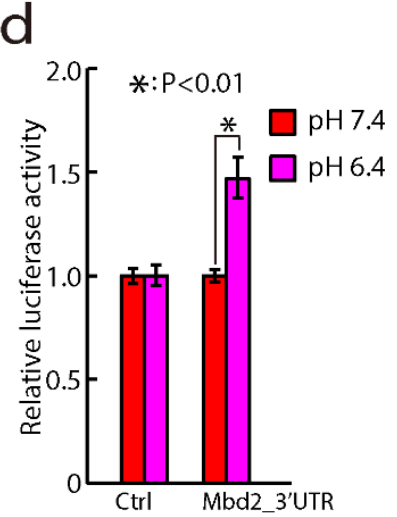

e

Dgcr8 KO_DO

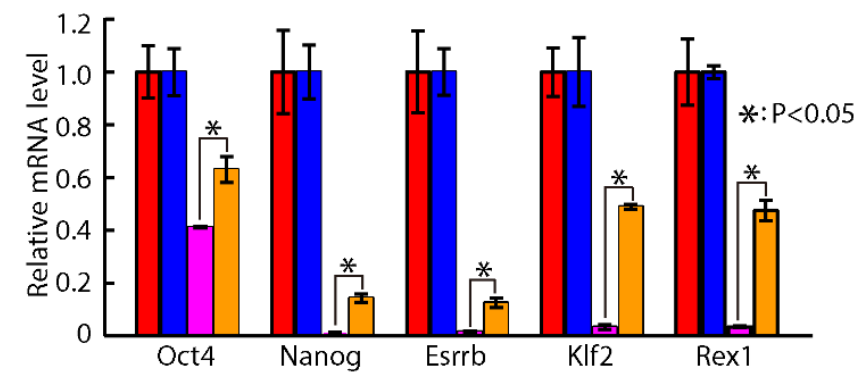

f

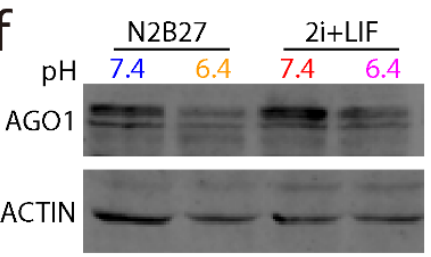

$*: P<0.05$

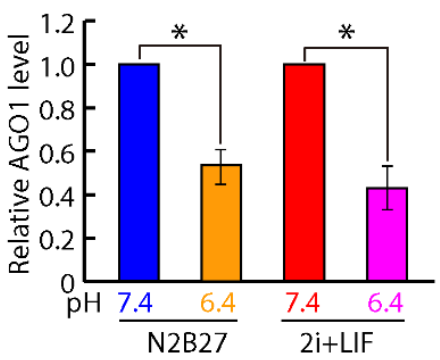

9

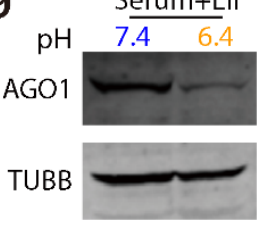

$*: P<0.05$

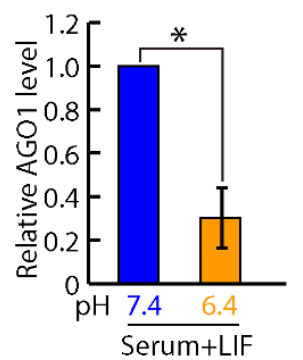

$\mathrm{h}$

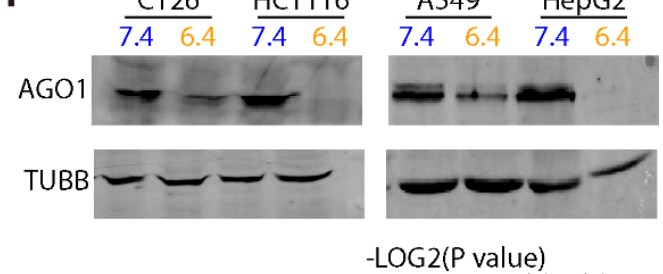
miR-93, miR-302a-d, miR-372

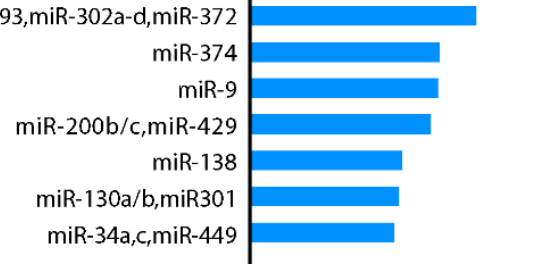




\section{Figure 7}

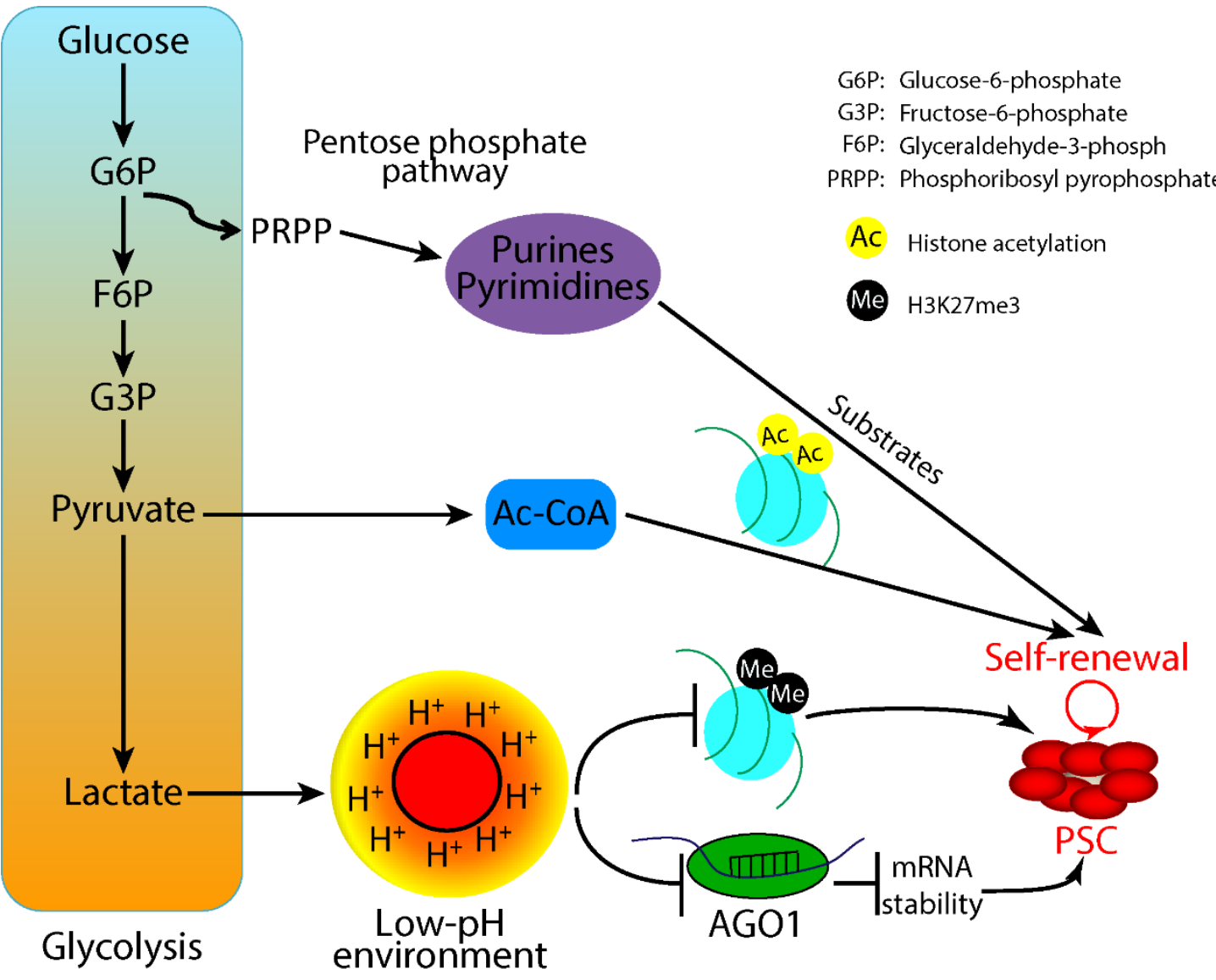




\section{SUPPLEMENTARY FIGURE LEGENDS}

Figure S1, Related to Figure 1. Low pH promotes the self-renewal and pluripotency of mouse ESCs. (a) MTT assay to test different $\mathrm{pH}$ conditions for maintaining pluripotency. OCT4-GFP-ires-Puro mESCs were exposed to N2B27 media at different $\mathrm{pH}$ for three days, and selected by $2 \mu \mathrm{g} / \mathrm{ml}$ puromycin in $2 \mathrm{i}$ media for two days. Shown are mean $\pm \mathrm{SD}, \mathrm{n}=4$. (b, c) FACS analysis of GFP positive portions of Rex1-GFP reporter cells in different culture conditions. (d) qRT-PCR analysis of pluripotency markers of low pH treated mESCs in Serum media; The Rpl7 gene was used as a control. Data were normalized to the mRNA level of Serum + LIF cultured mESCs. Shown are mean $\pm \mathrm{SD}, \mathrm{n}=2$. (e) $\mathrm{pH}$ value of media with or without HEPES buffer after growing ESCs for two days.

Figure S2, Related to Figure 2. Low $\mathrm{pH}$ treated mESCs are similar to naive mESCs cultured in $2 \mathrm{i}+\mathrm{LIF}$ at transcriptomic and epigenomic levels. (a) PCA analysis of global mRNA expression. (b) Heatmap of selected genes that are related to pluripotency and differentiation; (c) Heatmap of global miRNA expression. (d) Box plot showing pathway analysis for mESCs under normal $\mathrm{pH}$ and low-pH conditions. (e) ChIP-qPCR analysis of OCT4 binding at the promoter of pluripotency genes. (f) Screenshot of OCT4 binding of pluripotency genes Oct4, Sox2 and Nanog under different culture conditions; (g) Screenshot of H3K27me3 ChIP reads mapping to the promoter of Foxd1 and $L h x 5$ under different culture conditions.

Figure S3, Related to Figure 1 \& 2. Low pH plus LIF supports long term self-renewal of mouse ESCs. (a) Morphology of passage 2 and 5 ESC colonies. (b) qRT -PCR of pluripotency genes for passage 2 LIF pH 7.4 cells and passage 5 LIF pH 6.8 cells. The Rpl7 gene was used as a control. Data were normalized to the mRNA level of $2 \mathrm{i}+\mathrm{LIF}$ cultured mESCs. Shown are mean $\pm \mathrm{SD}, \mathrm{n}=2$.

Figure S4, Related to Figure 4. Generation of Eed knockout mouse ESCs. (a) 
Illustration of guide RNA (gRNA) sequences for knocking out Eed; location of primers used for genomic PCR confirming knockout of Eed are shown. (b) Genomic PCR of Eed knock out ESCs; (c) qRT -PCR amplification plot of $\beta$-actin and Eed in wild type and Eed knockout mouse ESCs. (d) qRT-PCR analysis of pluripotency markers in wildtype mESCs and Eed KO mouse ESCs differentiated for 2 days in N2B27 media without $2 \mathrm{i}$ and LIF. The $\beta$-actin gene was used as a control. Data were normalized to the mRNA level of $2 \mathrm{i}+\mathrm{LIF}$ cultured mESCs. Shown are mean $\pm \mathrm{SD}, \mathrm{n}=2$.

Figure S5, Related to Figure 5. Acidic $\mathrm{pH}$ stabilizes a large number of genes including many pluripotency genes in ESCs. (a) mRNA half-life in RNA-seq data and qRT-PCR analysis for random picked genes; Genes with consist mRNA half-life between RNAseq data and qRT-PCR analysis were labelled with " $\sqrt{ } "$. (b) Box plot of Log2 fold changes (pH6.4/pH7.4) of mRNA expression for genes with half-life increased or decreased > 2 fold in pH6.4 media. (c) Cumulative plot of Log2 fold changes (pH6.4/pH7.4) of mRNA expression for genes with half-life increased or decreased > 2 fold in pH 6.4 media.

Figure S6, Related to Figure 5. Acidic pH stabilizes ECM genes in cancer cells. (a) Morphology of human cancer cell lines HepG2 and U2OS at different pH; (b) Heatmap showing mRNA expression of epithelial (Cdh1) and mesenchymal markers in human cancer cell lines HepG2 and U2OS. (c) mRNA degradation plot of two representative ECM genes. The $\beta$-actin gene was used as a control. For each gene, data were normalized to U2OS cells cultured in each condition without actinomycin D treatment (0 hour). Shown are mean $\pm \mathrm{SD}, \mathrm{n}=2$. Actinomycin $\mathrm{D}$ was added $48 \mathrm{hr}$ after low $\mathrm{pH}$ treatment, and cells were collected at $0,2,4$, and $8 \mathrm{hr}$ after actinomycin D addition for RNA-seq.

Figure S7, Related to Figure 6. Low pH downregulates AGO1 protein expression and de-represses a subset of miRNA targets. (a) Venn diagrams showing the overlap between genes upregulated in Dgcr8 knockout mouse ESCs and genes with increased 
half-life in low pH treated mouse ESCs; (b) GSEA analysis showing the enrichment of upregulated genes in Dgcr8 knockout versus wild type ESCs in low pH treated ESCs; (c) Expression levels of miR-290/302 family of miRNAs in pH 6.4 and pH 7.4 ESCs from small RNA-seq. (d) Expression levels of miR-290/302 family of miRNAs in pH 6.4 and $\mathrm{pH}$ 7.4 ESCs from miRNA qPCR. Data were normalized to 7SK RNA and then to $2 \mathrm{i}+\mathrm{LIF}$ pH 7.4 ESCs. Shown are mean $\pm \mathrm{SD}, \mathrm{n}=2$. (e) Expression levels of Ago1 and Ago2 in different culture conditions from RNA-seq. Data were normalized to FPKM in $2 \mathrm{i}+\mathrm{LIF}$ pH 7.4 ESCs. Shown are mean $\pm \mathrm{SD}, \mathrm{n}=2$. (f) Western analysis AGO2 of mESCs in different culture conditions, The ACTIN was used as a loading control. Top panel: representative gel images. Bottom panel: quantification of AGO2 levels. Data were normalized to ACTIN then to $\mathrm{pH} 7.4$ media. Shown are mean $\pm \mathrm{SD}, \mathrm{n}=2$. 


\section{Supplementary Figure 1}
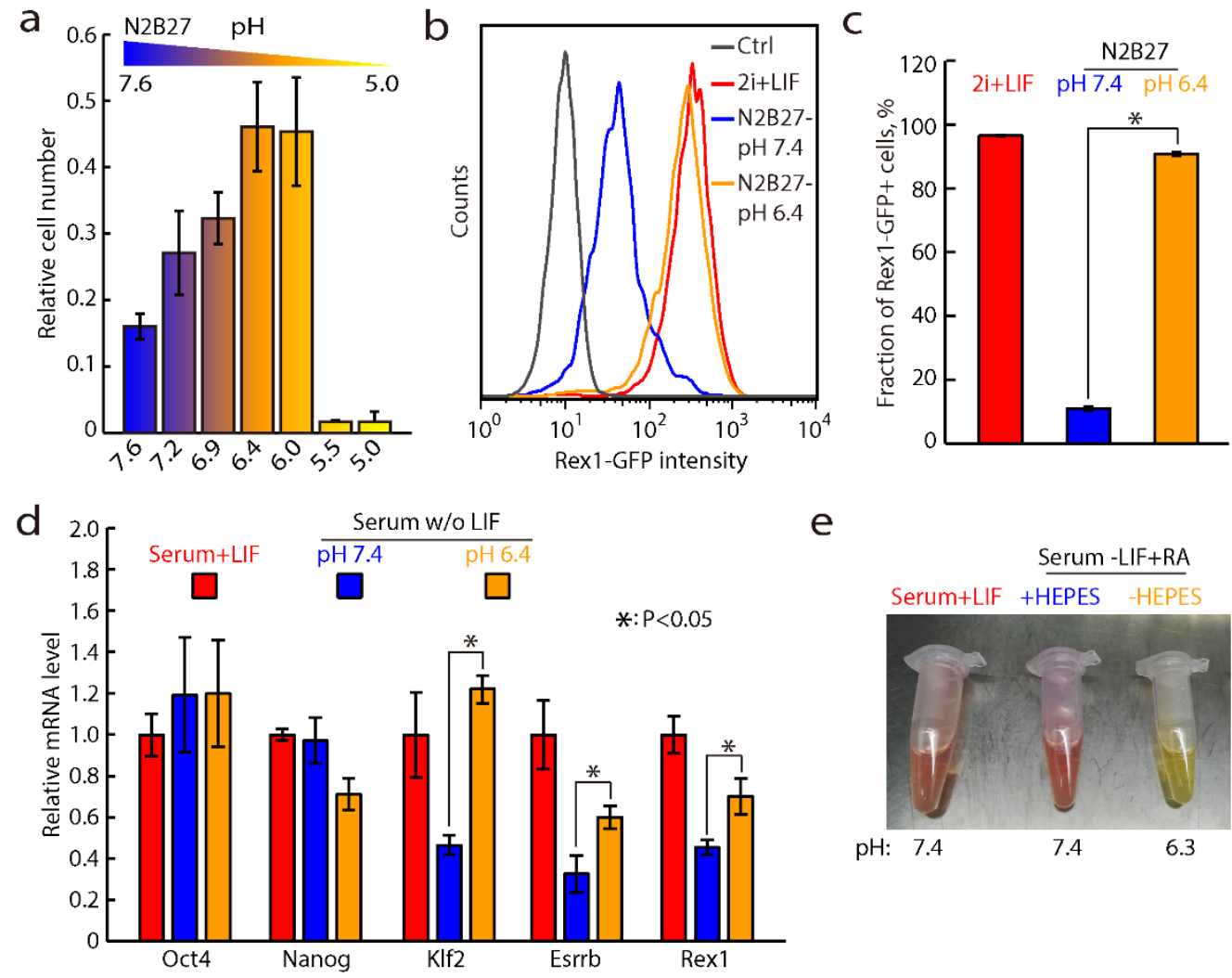


\section{Supplementary Figure 2}
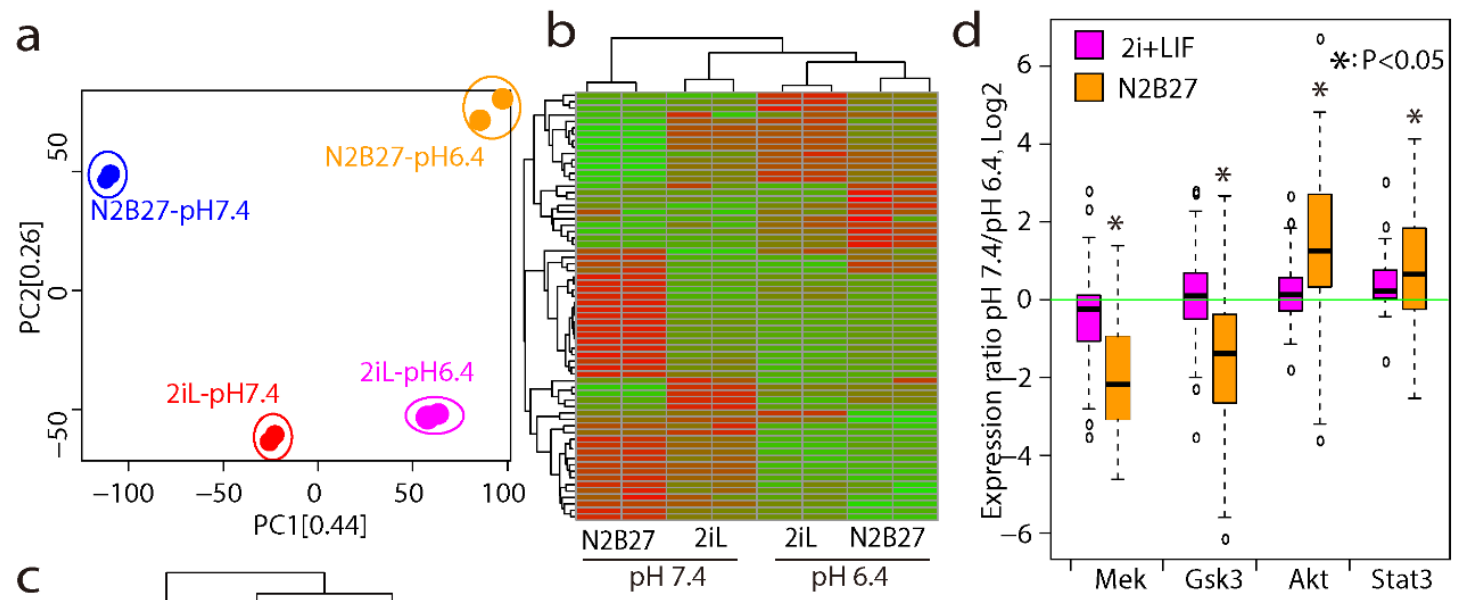

C

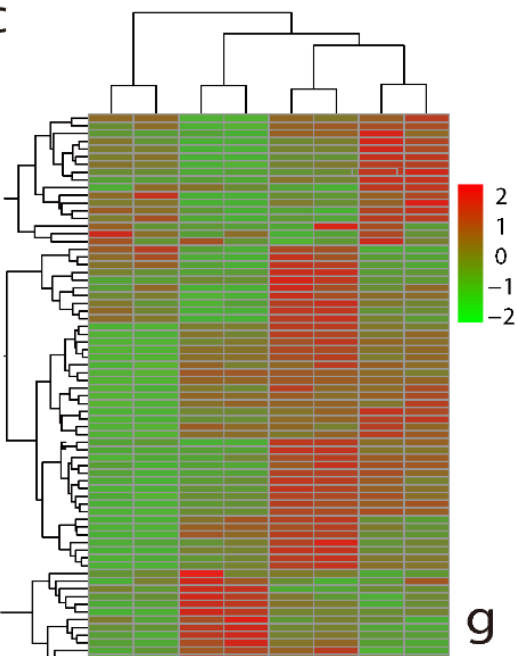

e

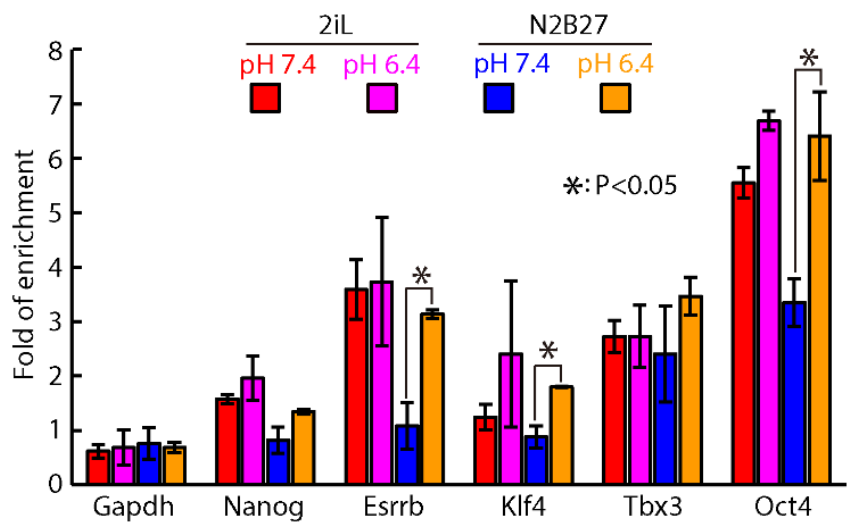

g

$5^{-}$

H3K27me3-CHIP

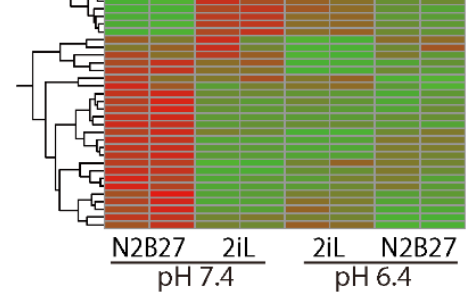

2iL pH7.4

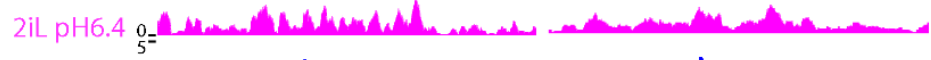
N2B27 pH7.4

f
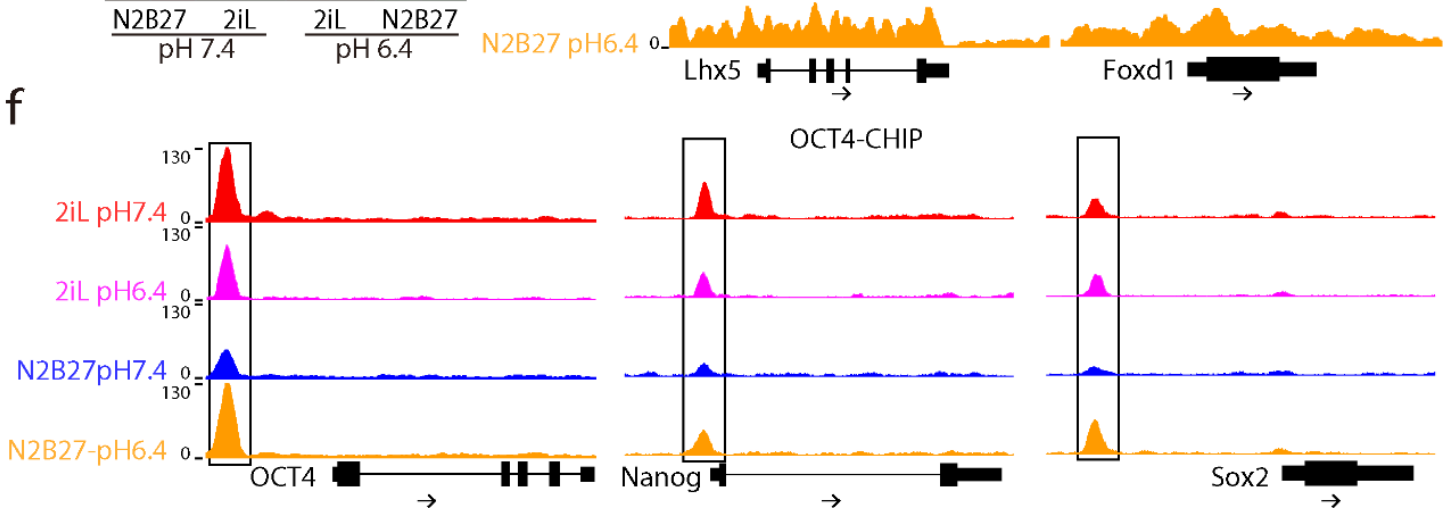


\section{Supplementary Figure 3}
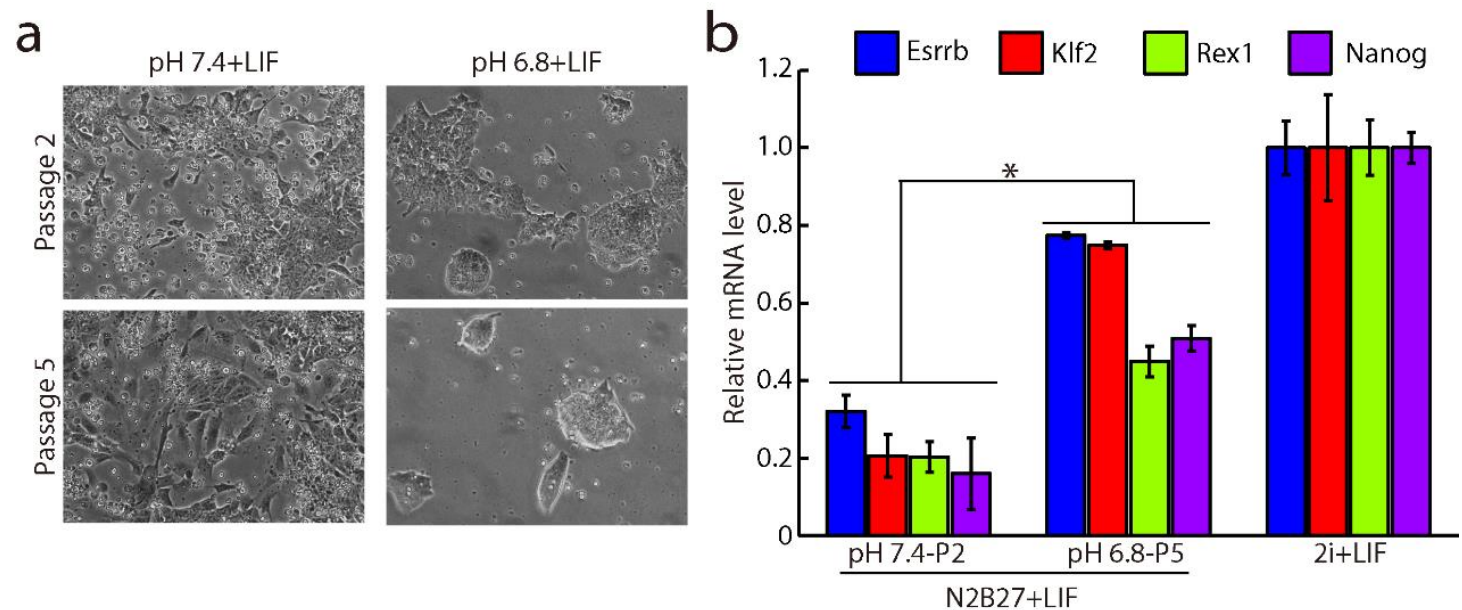


\section{Supplementary Figure 4}

a

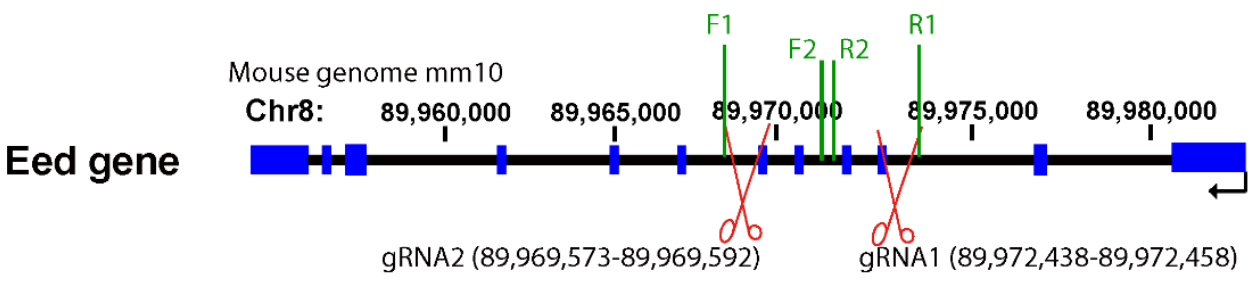

b

WT Eed KO $\mathrm{H} 2 \mathrm{O}$

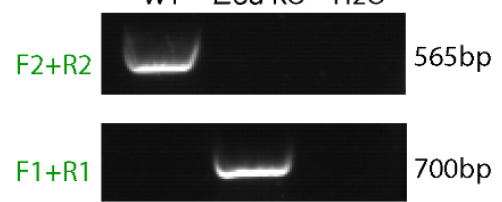

$\mathrm{d}$

WT_2iL $\square$ WT_N2B27

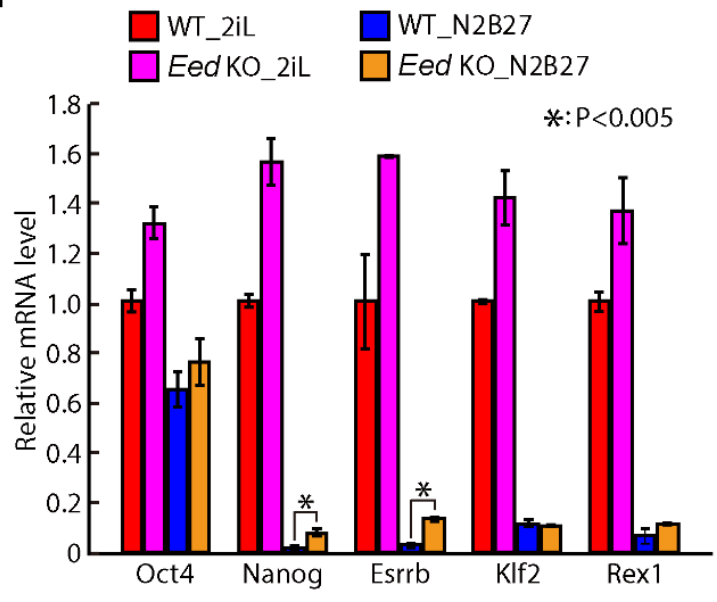

C

Amplification plot
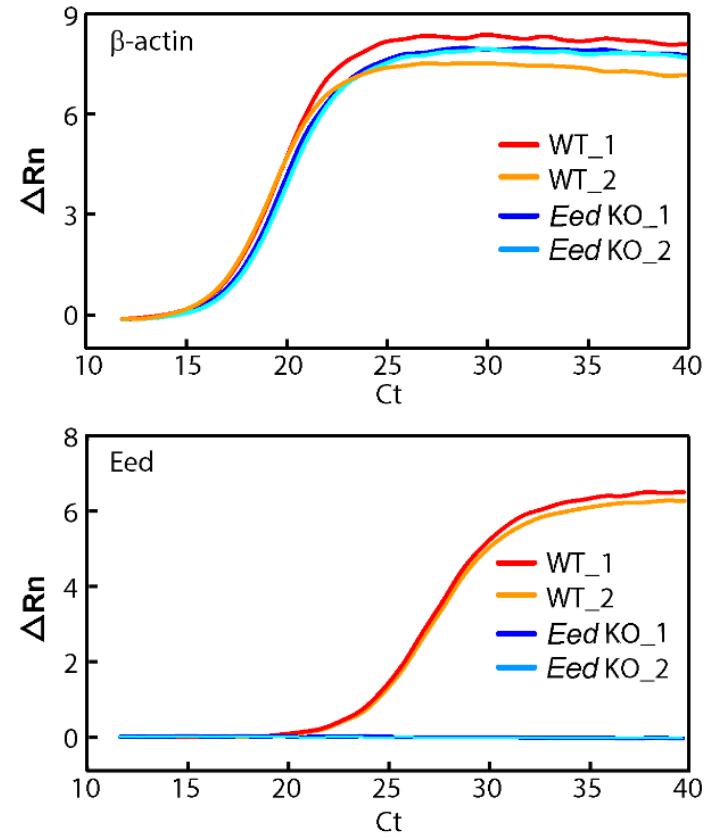


\section{Supplementary Figure 5}

a

\begin{tabular}{l|l|c|c|c|c|}
\hline & Seq-pH 7.4 & Seq-pH 6.4 & qPCR-pH 7.4 & qPCR-pH 6.4 \\
\cline { 2 - 6 } & Atp1b1 & 3.02 & 6.23 & 2.87 & 4.38 \\
\cline { 2 - 6 } & Atp9a & 10.83 & 24.00 & 9.94 & 24.00 \\
\cline { 2 - 6 } & Fbxo15 & 4.49 & 5.96 & 2.67 & 3.15 \\
\cline { 2 - 6 } & Pim1 & 2.04 & 2.78 & 1.29 & 1.74 \\
\cline { 2 - 6 } & Relb & 3.67 & 5.73 & 1.94 & 3.37 \\
\cline { 2 - 6 } & Stat3 & 6.37 & 8.75 & 2.41 & 3.91 \\
\cline { 2 - 6 } & Nfkb2 & 6.27 & 24.00 & 4.10 & 8.34 \\
\cline { 2 - 6 } & Nr0b1 & 3.54 & 4.54 & 3.67 & 9.50 \\
\cline { 2 - 6 } & Ncoa3 & 4.67 & 4.37 & 1.73 & 1.62 \\
\cline { 2 - 6 } & Nr5A2 & 1.92 & 2.01 & 1.26 & 0.96 \\
\cline { 2 - 6 } & Zfp42 & 1.89 & 2.14 & 1.19 & 1.13 \\
\hline & Gadd45b & 2.62 & 5.73 & 1.04 & 1.13 \\
\hline & 11.57 & 22.71 & 24.00 & 13.30 \\
\hline
\end{tabular}

b

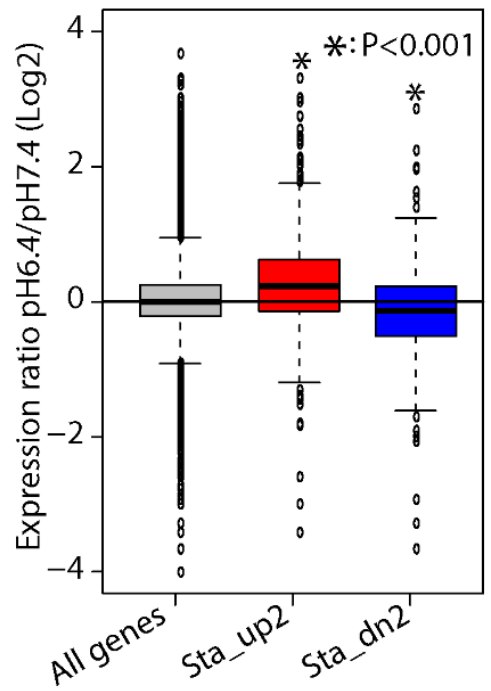

C

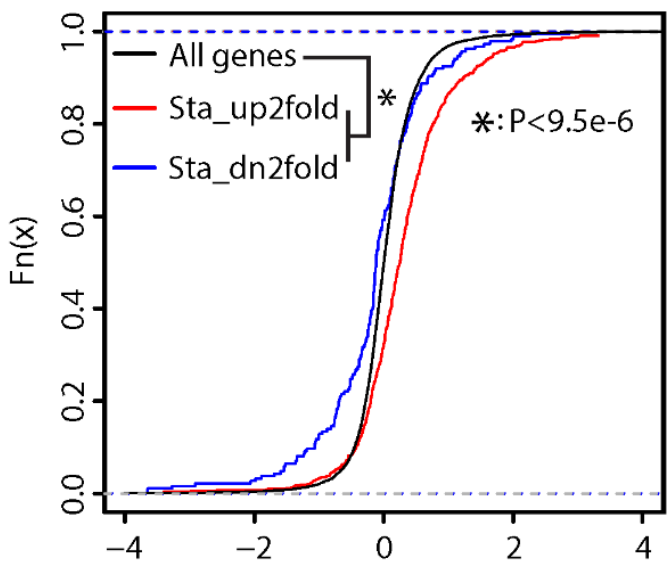

Expression ratio $\mathrm{pH} 6.4 / \mathrm{pH} 7.4, \log 2$ 


\section{Supplementary Figure 6}

a
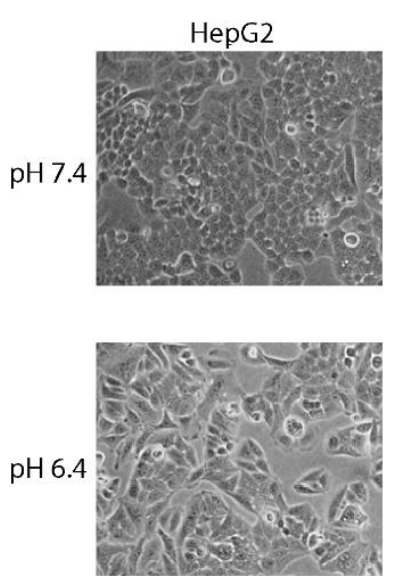

C

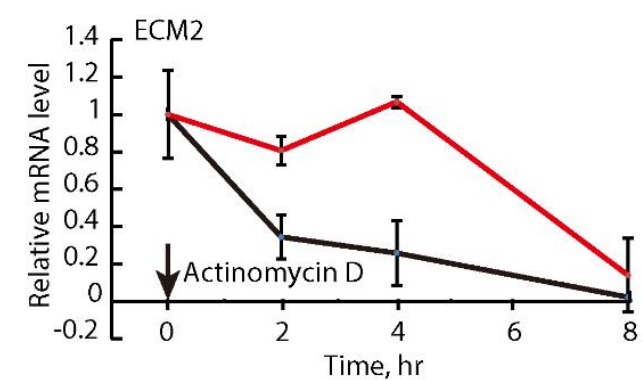

b
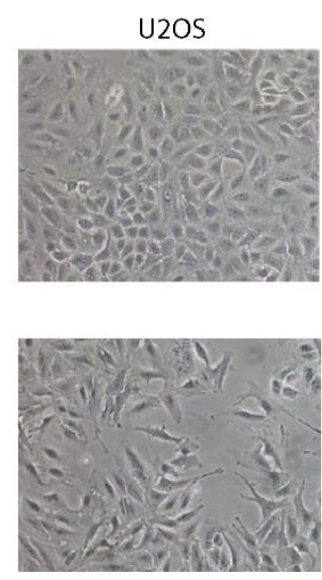

$\mathrm{pH} 7.4$

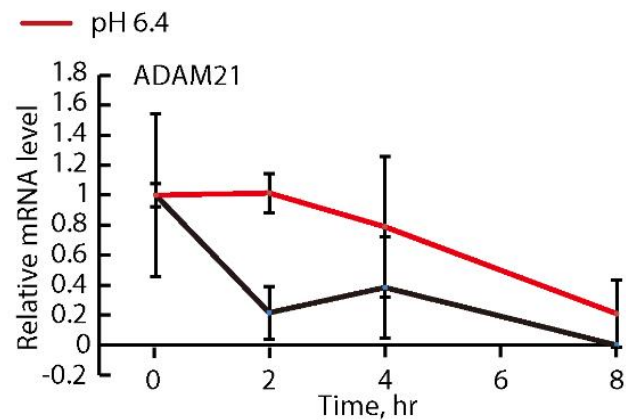




\section{Supplementary Figure 7}

a

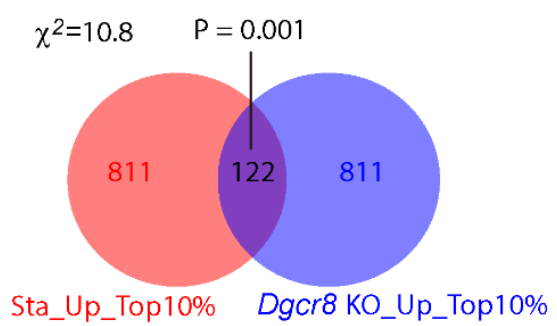

C

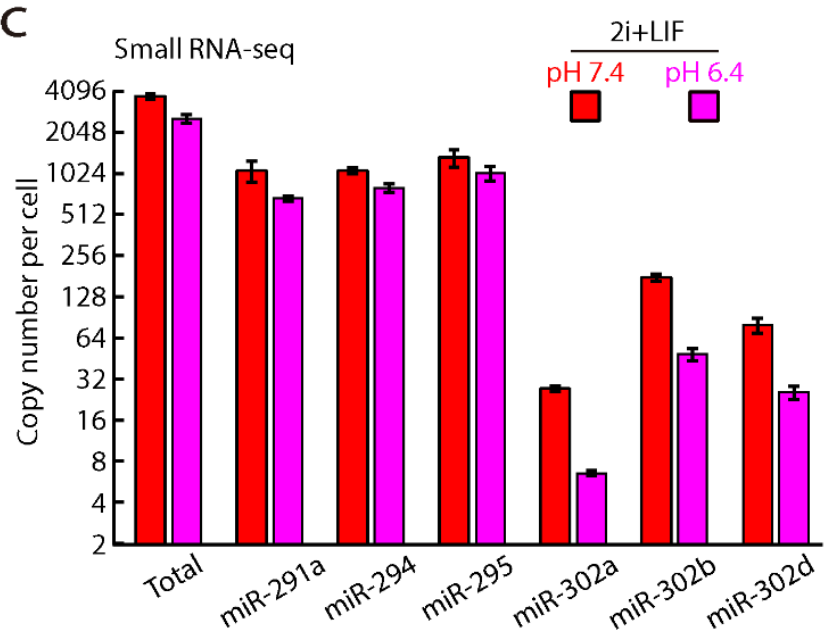

d

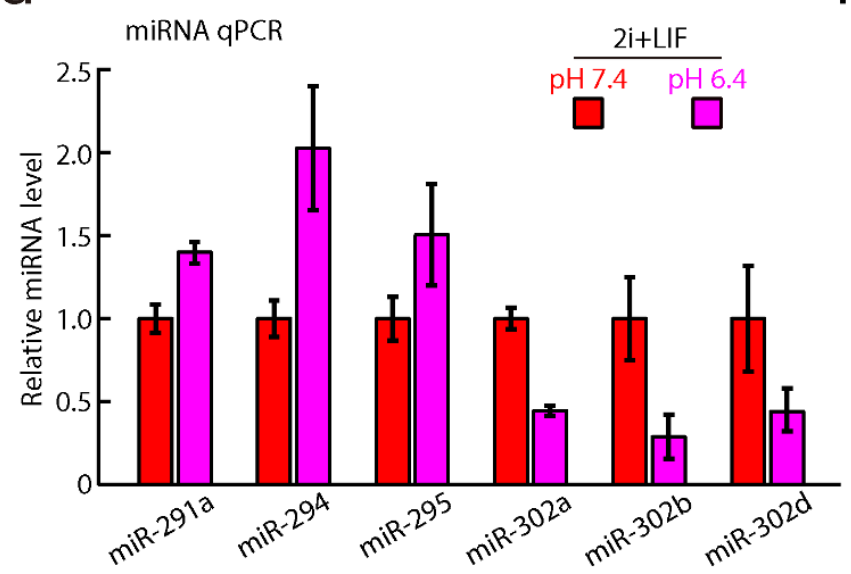

b

Gene Set Enrichment Analysis (GSEA)

Dgcr8 KO up_Top10\% geneset in pH6.4 treatment

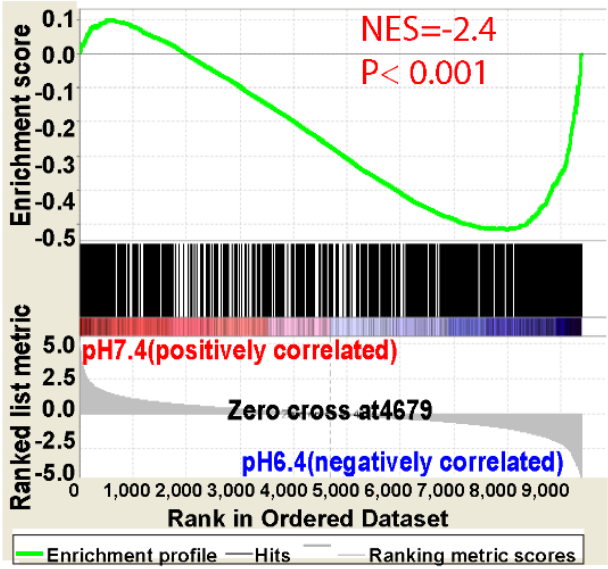

e

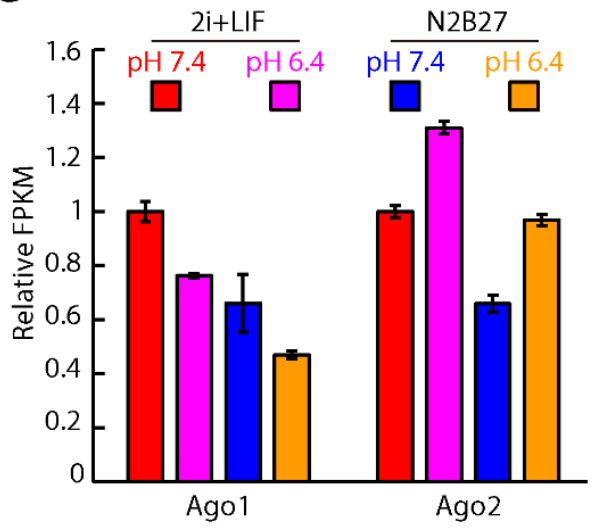

f
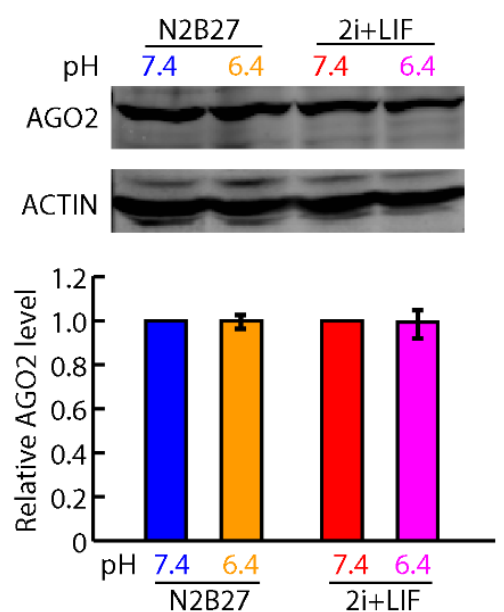